\title{
COMPUTER SIMULATION OF THE EMITTANCE GROWTH DUE TO NOISE IN LARGE HADRON COLLIDERS
}

\author{
V. LEBEDEV* \\ Superconducting Super Collider Laboratory, ${ }^{\dagger}$ \\ 2550 Beckleymeade Ave., Dallas, TX 75237 \\ (Received 28 April 1993; in final form 10 February 1994)
}

\begin{abstract}
The problem of emittance growth due to random fluctuations of the magnetic field in a hadron collider is considered. The results of computer simulations are compared with the analytical theory developed earlier. A good agreement was found between the analytical theory predictions and the computer simulations for the collider tunes located far enough from high-order betatron resonances. The dependence of the emittance growth rate on noise spectral density, beam separation at the interaction point (IP), and the value of beam separation at long range collisions is studied. The results are applicable to the Superconducting Super Collider (SSC).
\end{abstract}

KEY WORD: Emittance growth, noise, hadron, colliders, particle dynamics, storage rings

\section{INTRODUCTION}

This paper relates to a preceding article. ${ }^{1}$ The material was divided into two papers because of its length; hopefully, this has resulted in better clarity and simplicity.

\section{BASIC COMPUTER MODEL AND ITS RESULTS}

To test the predictions of the theory developed in the previous article and to study other effects not included therein, we have carried out numerical simulations with the particle motion being influenced by external noise and the feedback system. The simulations were done with the basic computer model and its modifications. The results are presented in the sections that follow.

\footnotetext{
* Visiting from the Budker Institute of Nuclear Physics, Novosibirsk, Russia.

$\dagger$ Operated by the Universities Research Association, Inc., for the U.S. Department of Energy under Contract No. DE-AC35-89ER40486.
} 


\subsection{Basic Computer Model}

The model simulates the two-dimensional (four-dimensional phase space) motion of an ensemble of particles in a beam having initially a Gaussian density distribution. It takes into account collisions of the particles with a round counter-rotating beam also having Gaussian distribution, whose rms radius is assumed to remain constant in the course of the tracking. This target beam behaves like one macroparticle and we will call it the rigid beam. In contrast, we will call the beam consisting of particles the soft beam. The effects of the ring nonlinearity, synchrotron motion, and finite bunch length were neglected in these simulations. All runs were performed for one IP in the ring.

Mathematically, the particle motion in the storage ring has been modeled as a sequence of mappings governing evolution of the phase space coordinates $x, p_{x}$ and $y$, $p_{y}$ defined for each degree of freedom, in accordance with Equation (1) of Reference 1 . For convenience, we scaled all the coordinates and momenta by the rms radius $\sigma$ at the IP.

First, the phase variables of each particle were transformed with the one-turn linear map,

$$
\left(\begin{array}{c}
x_{j}^{\prime} \\
p_{x j}^{\prime}
\end{array}\right)=\left(\begin{array}{cc}
\cos 2 \pi \nu_{x} & \sin 2 \pi \nu_{x} \\
-\sin 2 \pi \nu_{x} & \cos 2 \pi \nu_{x}
\end{array}\right)\left(\begin{array}{c}
x_{j} \\
p_{x j}
\end{array}\right), \quad x_{j}, p_{x j}, \nu_{x} \leftrightarrow y_{j}, p_{y j}, \nu_{y},
$$

that describes the particle motion in the lattice. Here $j=1,2, \ldots N_{\text {particle }}$ enumerates particles.

The mapping, Equation (1), was followed by three subsequent transformations that correspond to a beam-beam collision, a noise perturbation, and a feedback kick.

The head-on beam-beam interaction of a particle with a rigid beam was modeled by the following kick, ${ }^{2}$

$$
p_{x j}^{\prime}=p_{x j}+\frac{8 \pi \xi x_{j}}{r_{j}^{2}}\left(1-\exp \left(-\frac{r_{j}^{2}}{2}\right)\right), \quad x_{j}, p_{x j} \leftrightarrow y_{j}, p_{y j},
$$

where $r_{j}^{2}=x_{j}^{2}+y_{j}^{2}$ and $\xi$ is the interaction parameter (head-on beam-beam tune shift) given by Equation (16) of Reference 1 .

The cumulative effect of the noise in all of the ring magnets on one-turn was modeled by the following transformation:

$$
\begin{array}{ll}
p_{x j}^{\prime}=p_{x j}+\frac{\Delta}{\sqrt{2}} \zeta^{\left(p_{x}\right)}, & x_{j}^{\prime}=x_{j}+\frac{\Delta}{\sqrt{2}} \zeta^{(x)}, \\
p_{y j}^{\prime}=p_{y j}+\frac{\Delta}{\sqrt{2}} \zeta^{\left(p_{y}\right)}, & y_{j}^{\prime}=y_{j}+\frac{\Delta}{\sqrt{2}} \zeta^{(y)},
\end{array}
$$

where $\Delta$ characterizes the strength of the noise, and $\zeta^{\left(p_{x}\right)}, \zeta^{\left(p_{y}\right)}, \zeta^{(x)}$ and $\zeta^{(y)}$ are random numbers having a Gaussian distribution with dispersion equal to 1 . These 
numbers were generated each turn independently for each of the directions in the phase space. It is assumed in Equation (3) that the beam experiences the noise kicks from many magnets evenly distributed around the ring. In this case, because of betatron oscillations and independence of kicks from different magnets, the sum of kicks averaged over one turn yields equal increments for the coordinates and momenta.

The presence of the feedback system in the storage ring was simulated in accordance with the model of Section 4.1 of Reference 1 . In this model the feedback system is located next to the interaction region, and

$$
\begin{aligned}
& p_{x j}^{\prime}=p_{x j}-g \bar{p}_{x}, \\
& p_{y j}^{\prime}=p_{y j}-g \bar{p}_{y},
\end{aligned}
$$

where $\bar{p}_{x}, \bar{p}_{y}$ are the averaged momenta of beam,

$$
\bar{p}_{x}=\frac{1}{N_{\text {particle }}} \sum_{j=1}^{N_{\text {particle }}} p_{x j}, \quad \bar{p}_{y}=\frac{1}{N_{\text {particle }}} \sum_{j=1}^{N_{\text {particle }}} p_{y j} .
$$

As can be seen from Equations (3) and (4) all particles experience the same kick at the noise and damping transformations.

Emittance of the beam has been computed with the use of the following formula:

$$
\varepsilon=\frac{1}{4 N_{\text {particle }}} \sum_{j=1}^{N_{\text {particle }}}\left(\left(x_{j}-\bar{x}\right)^{2}+\left(y_{j}-\bar{y}\right)^{2}+\left(p_{x j}-\bar{p}_{x}\right)^{2}+\left(p_{y j}-\bar{p}_{y}\right)^{2}\right) .
$$

This definition of $\varepsilon$ allows one to avoid spurious variations of the emittance related to coherent beam motion.

We used as many as 5000-15000 particles to prevent the stochastic cooling effect which could mask the emittance growth in the case of a small number of particles. One can estimate the dimensionless stochastic cooling decrement with the help of the formula $^{3}$

$$
\gamma_{\text {stoch }} \approx \frac{\min (g, \Delta \nu / 2)}{2 N_{\text {particle }}},
$$

whose predictions have a very good agreement with simulation results in the absence of noise. In our simulations, we specially chose the parameters in order to make the decrements of cooling much smaller than the observed emittance growth rate.

To improve the accuracy of the simulations we ran the code several times (usually three) with different seeds of random numbers $\zeta$ and the initial particle distributions. The emittance growth was averaged over runs, and the variance was estimated. Multiple runs increased the simulation accuracy and allowed us to estimate the simulation errors. 
For comparison of the simulation results with theory for the white noise case, we used Equations (15), (19), and (49) of Reference 1. From these equations it follows that the emittance increase per turn is equal to

$$
\frac{d \varepsilon}{d n}=A \frac{\xi^{2} \Delta^{2}}{2 g^{2}}
$$

where $A \approx(0.17 \cdot 4 \pi)^{2} \approx 4.5$, as follows from theory, developed in Reference 1 , Section 4.2, Equations (17) and (49). (See also Appendix A herein.) However, taking into account the simplified nature of the theory, we consider $\mathrm{A}$ as a fitting parameter and determine its value so as to obtain the best agreement with the simulations.

\subsection{Simulations Results for the Basic Model}

Examples of the emittance growth in the vicinity of resonances $3 / 4$ and $5 / 6$ calculated with the use of the basic model are shown in Figures 1 and 2. One can see that far from resonances the emittance grows linearly with time. Oscillations of the emittance during the first two or three hundred turns are associated with a transition process caused by the fact that the initial distribution function (a Gaussian) is not an equilibrium one if beam-beam interaction is taken into account. Initial oscillations in the emittance reflect a process of restructuring of the initial distribution of the beam. As can be seen, the intensity and time of transition increase at the resonance.

To test the accuracy of the estimates presented in Section 4.2 of Reference 1, we also performed numerical simulations for an ideally linear model with given tune spread. In this model, the mapping Equation (2) has been eliminated; instead, each particle has been described by a constant tune determined by its initial amplitude $a=\sqrt{x^{2}+y^{2}+p_{x}^{2}+p_{y}^{2}}$. The tune shift dependence $\delta \nu(r)$ has been calculated using the first-order term of the perturbation theory ${ }^{a}$

$$
\delta \nu(a)=\frac{4 \xi}{a^{2}}\left(1-I_{0}\left(\frac{a^{2}}{4}\right) \exp \left(-\frac{a^{2}}{4}\right)\right),
$$

where $I_{0}$ is the zero-order modified Bessel function. This case differs in many respects from the nonlinear model because each particle represents a linear oscillator so that nonlinear resonances are eliminated from the particle dynamics.

The calculated emittance growth rates for linear and nonlinear models are presented in Figure 3 for the first 800 turns. One can see that for $\xi<0.03$ both models are in good agreement with each other and with the theoretical formula Equation (8). The difference between models arises at higher values of $\xi,(\xi \geq 0.03)$ when the nonlinear effects somewhat amplify the external noise. The saturation of the emittance growth rate in the linear model for $\xi>g$ is associated with the fact that it reaches its maximum value equal to the growth rate without the feedback system.

\footnotetext{
${ }^{a}$ Although Equation (9) is applicable only for one-dimensional motion and its use is not correct in the two-dimensional case, we used it to simplify simulations. This is justified because the rms tune spreads in both cases are very close (see Appendix A).
} 


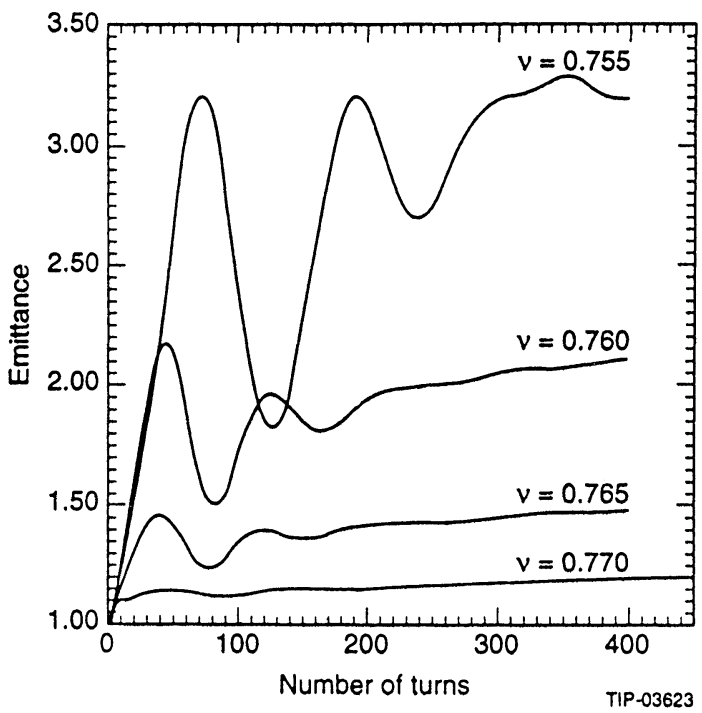

FIGURE 1: The dependence of emittance on time for different betatron tunes in the vicinity of a resonance $3 / 4 ; \xi=0.03, \Delta=0.05, g=0.2, \nu_{x}=\nu_{y}=\nu$.

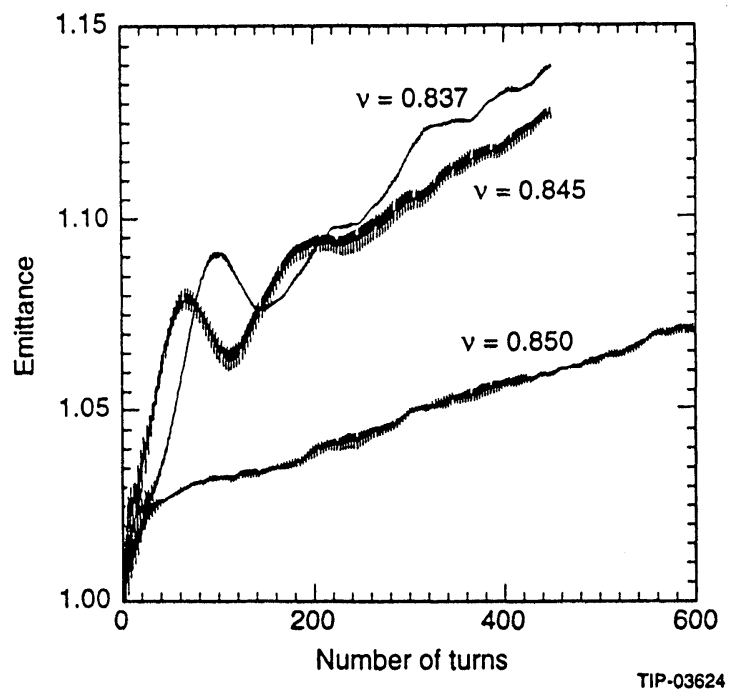

FIGURE 2: The dependence of emittance on time for different betatron tunes in the vicinity of a resonance $5 / 6 ; \xi=0.03, \Delta=0.05, g=0.2, \nu_{x}=\nu_{y}=\nu$. 


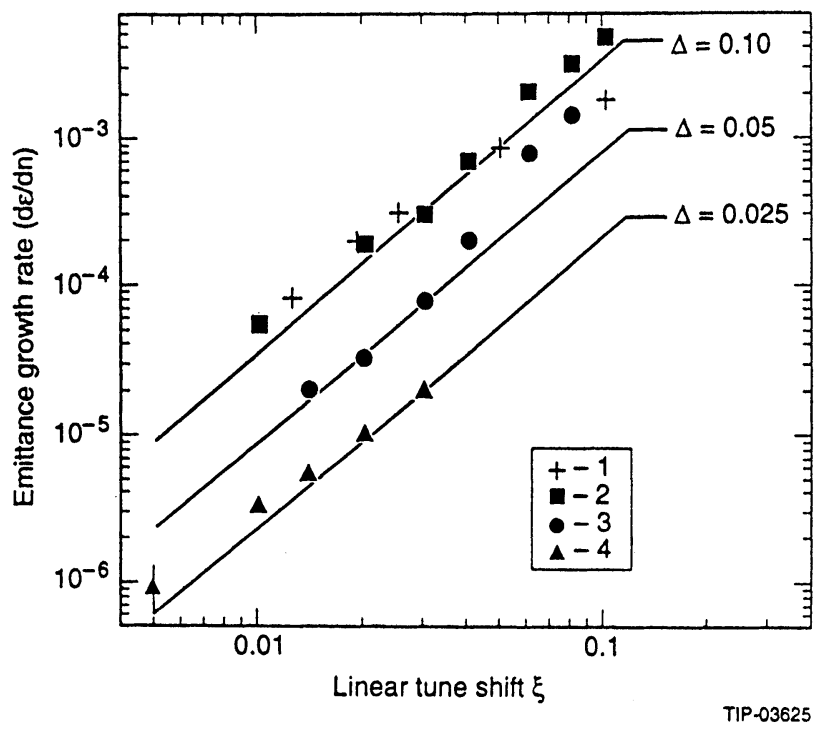

FIGURE 3: The dependence of the emittance growth rate on the beam-beam tune shift $\xi$ for different values of noise $\Delta ; g=0.2, \nu=0.78$. The lines are plotted using Equation (8) with $A=3.0$. Number of turns is equal to 800. In the linear model (1) $\Delta=0.1$. In the non-linear models (2), (3), and (4) $\Delta=0.1,0.05$, and 0.025 respectively.

Figure 4 shows the dependence of the emittance growth versus the amplitude of the noise $\Delta$ for the first 5000 turns. As can be seen from this figure, for larger values of $\Delta(\Delta>0.3)$ the emittance growth rate diverges from the theoretical curve. This can be understood if one takes into account that at large amplitudes of coherent oscillations comparable with the beam size, the beam separation at the IP would result in smaller betatron tune spread and, consequently, smaller emittance growth.

Figure 5 shows the dependence of the emittance growth rate on the dimensionless gain of the feedback system $g$. The emittance growth is calculated for the first 800 turns. Like Figure 3, Figure 5 shows that dependence saturates for $g<\xi$, where the emittance growth rate is limited by its value without the feedback system.

To understand why in Figure 3 the value $\xi \approx 0.03$ is limited by nonlinear interaction, we scanned betatron tunes in the vicinity of the tune ${ }^{b} \nu_{x}=\nu_{y}=0.78$ which was used in the previous figures. The results of this simulation are shown in Figure 6. One can see a strong influence of resonances $3 / 4$ and $5 / 6$ on the emittance growth rate. The resonances $8 / 10$ and $11 / 14$ can hardly be seen. This figure clearly demonstrates that

\footnotetext{
${ }^{b}$ This is the fractional part of the baseline SSC tune suggested in Reference 4. Different tunes are now being considered, but because the final decision has not been made to change it, we will consider it as the SSC tune. (This really does not limit the developed theory and estimations.)
} 


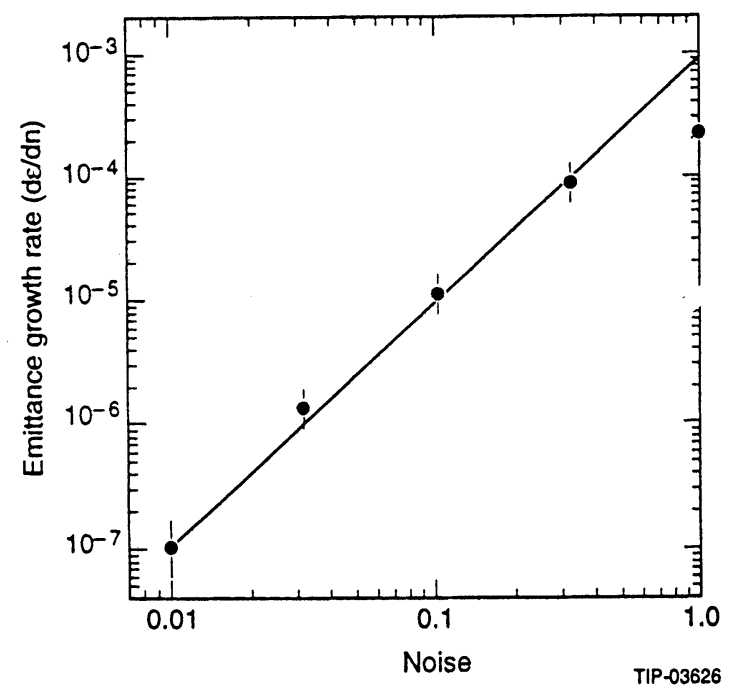

FIGURE 4: The dependence of the emittance growth rate on the kick value $\Delta ; g=0.2, \xi=0.005, \nu=0.78$. The line is plotted using Equation (8) with $A=3.5$. Number of turns is equal to 5000 .

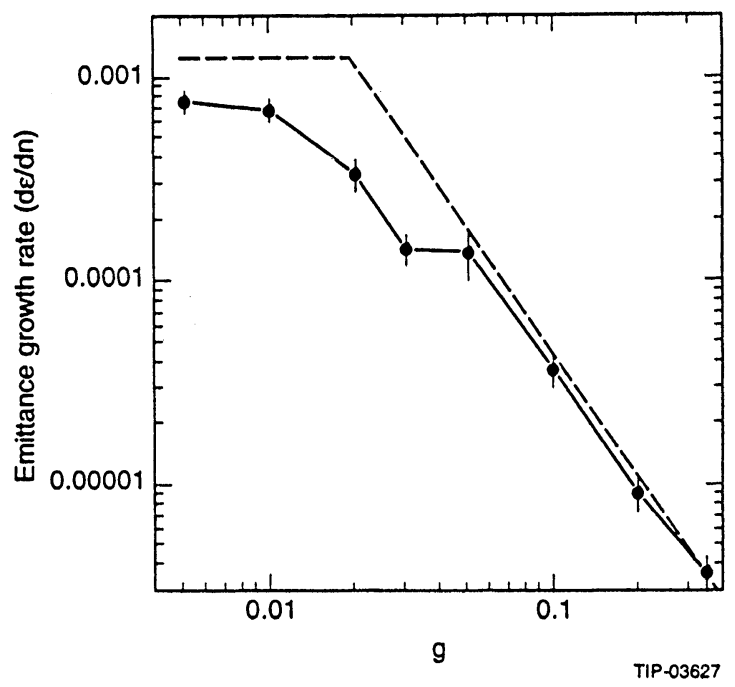

FIGURE 5: The dependence of the emittance growth rate on the dimensionless gain $g$ of the feedback system; $\Delta=0.05, \xi=0.01, \nu=0.78$. The dashed line is plotted using Equation (8) with $A=3.0$. The number of turns is equal to 800 . 


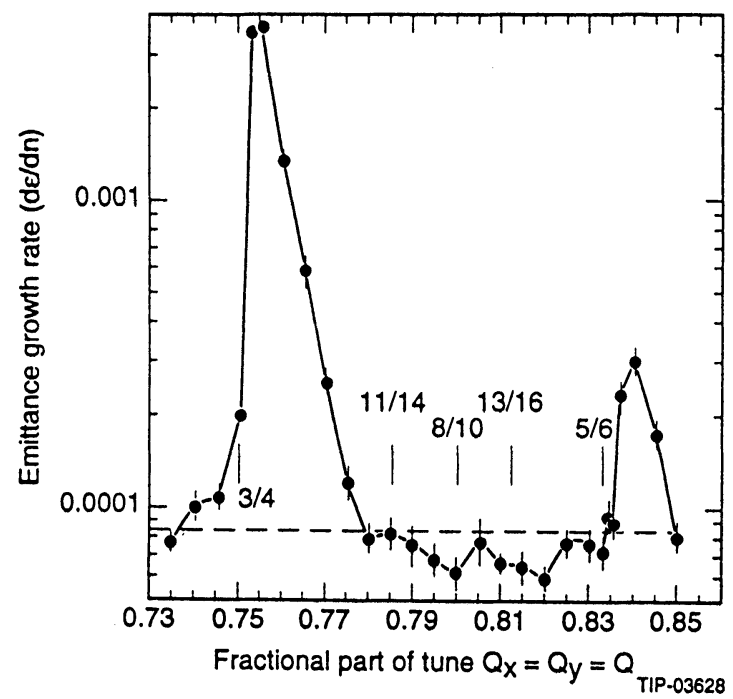

FIGURE 6: The dependence of the emittance growth rate on the tune $\nu ; g=0.2, \Delta=0.05, \xi=0.03$. The dashed line is plotted using Equation (8) with $A=3.0$. The number of turns is equal to 800 .

the additional increase in the emittance growth rate for $\nu_{x}=\nu_{y}=0.78$ and $\xi \geq 0.03$ can be explained by the influence of the strong resonance $3 / 4$. This shows that the nonlinearity of motion (which is not included in the first model) strongly affects the particle motion only in the case of sufficiently high values of $\xi$. For low values of $\xi$ and collider tunes located not too close to a resonance, one can neglect the influence of nonlinear resonances on particle motion. In this case, as can be seen from Figures 3 and 5, the dependence of the emittance growth rate on $\xi$ is given by $d \varepsilon / d t \propto(\xi / g)^{2}$ for $\xi<g$ and $d \varepsilon / d t=$ const for $\xi>g$ in accordance with our theoretical model.

\section{COHERENT BEAM-BEAM EFFECTS IN THE PRESENCE OF EXTERNAL NOISE}

In the previous section we presented the results of the simulations that assumed that the rigid beam was at rest. In reality, however, both beams will undergo the influence of the noise, whose effect on the rigid beam would be to excite its coherent oscillations similar to that of the soft beam. These coherent oscillations of the rigid beam would increase the emittance growth of the soft beam because, in addition to the random kicks produced by magnets, the soft beam will also be perturbed at the IPs by random electromagnetic fields rising from the coherent motion of the rigid beam.

Similar to the case without coherent beam-beam effects discussed before, we can 
distinguish two cases: without and with strong damping of the transverse feedback system.

In the first case, without strong damping, the presence of the coherent motion does not strongly change the emittance growth rate. A simple analytical model was developed in Reference 2 in the hard-bunch approximation (see Appendix B) where the bunches were considered as rigid ones and only dipole interaction was taken into account. In this case the emittance growth rate under external noise is equal to

$$
\frac{d \varepsilon}{d t}=\left(\frac{d \varepsilon}{d t}\right)_{0}\left[1+\frac{(4 \pi \xi)^{2}}{2 \sin (2 \pi \nu)\left(\sin (2 \pi \nu)-4 \pi \xi \cos (2 \pi \nu)-(4 \pi \xi)^{2} \sin (2 \pi \nu)\right)}\right]
$$

where $(d \varepsilon / d t)_{0}$ is the emittance growth rate in the absence of the coherent beam-beam effects determined by Equation (19) of Reference 1. The second term in the square brackets corresponds to the contribution of the coherent dipole interaction. As can be seen in the vicinity of integer and half-integer resonances where

$$
\sin (2 \pi \nu)\left(\sin (2 \pi \nu)-4 \pi \xi \cos (2 \pi \nu)-(4 \pi \xi)^{2} \sin (2 \pi \nu)\right) \leq 0,
$$

the beam motion is unstable ${ }^{5}$, and the emittance growth rate is larger near these unstable regions. Nevertheless, the beam coherent motion and emittance growth rate are not changed significantly far from half-integer resonances $(\delta \nu>\xi)$ if the $\xi$ value is small.

If we take into account higher-order nonlinear resonances, we also obtain the amplification of emittance growth rate at these resonances. The resonance width will also be of the order of $\xi$, and a resonance influence will also be small far from the resonances. As can be seen in the absence of the feedback system and small $\xi$ value, the coherent beam-beam effects will have a produce negligible effect on the emittance growth. In particular, we expect that their influence will be very small on the SSC because of small $\xi$ value.

In the second case, when the strong feedback system suppresses the betatron motion, the situation is slightly different. To understand the feedback influence we carried out direct numerical simulations of the emittance growth rate in the strong-strong beam approximation. In this case, as in the basic model, one bunch consists of many particles (up to 15300) and the other one is rigid but is allowed the two-dimensional betatron motion of this bunch. As before, the rigid bunch has a Gaussian density distribution, and its size does not change in time. Both bunches are influenced by independent external noises and the damping systems. The motion of the beams is determined by Equations (1-4) where in Equation (2) we consider that $r$ is the distance between a particle of the soft bunch and the centroid of the rigid bunch for soft beam particles and $r$ is the distance between centroids of bunches for the rigid beam.

The results of these simulations are shown in Figure 7. The simulations confirm that, similar to the case without a feedback system, there is no change of the dipole coherent betatron motion by counter-rotating beam fields; nevertheless, the resultant emittance growth rate is significantly larger. One can see that for $g=0.2$ the dependence of 


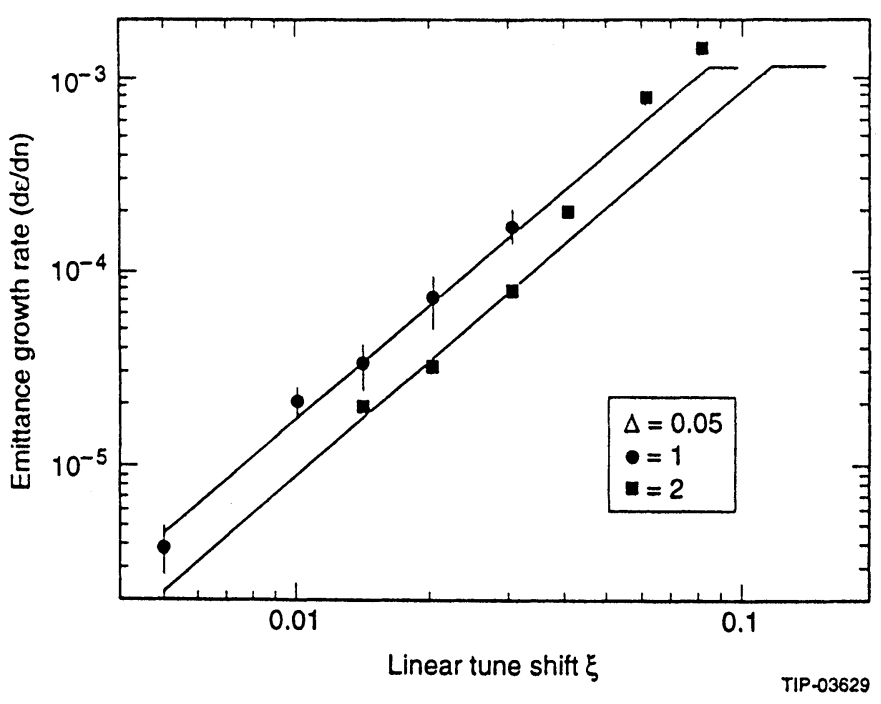

FIGURE 7: The dependence of the emittance growth rate on the beam-beam tune shift $\xi$ with (upper curve) and without (lower curve) a coherent motion of the rigid bunch; $g=0.2, \Delta=0.05, \nu=0.78$. The number of turns is equal to 800 .

the emittance growth rate on $\xi$ is similar to the one described above, but its value is doubled. Thus for $g=0.2$ these calculations redetermined the value of the constant $A$ in Equation (6) to be equal to $\approx 7$ in the presence of a coherent beam-beam motion instead of $\approx 3.5$ for the case without it.

It seems plausible that this additional increase in the emittance growth rate is bound up with the influence of higher-order nonlinearities. Because the dipole motion of bunches is strongly suppressed by the feedback system, the emittance growth due to dipole kicks is also strongly suppressed. The effect of the high-order nonlinearities is not damped by the feedback system, and their influence will become more and more important with increasing damping. We do not have a clear theoretical picture of the influence of the high-order nonlinearities, but the simplest estimate considered below is in reasonable agreement with numerical simulations and clarifies the general picture.

We estimate the parametric excitation of particle motion by fields of a rigid counter bunch. The excitation results from the change of the counter bunch focusing strength due to its transverse motion, which is of a random character. In the first approximation, the value of angle kick is about:

$$
\delta p=\frac{8 \pi \xi}{r}\left(1-\exp \left(-\frac{r^{2}}{2}\right)\right) \approx 4 \pi \xi\left(x-x_{c}-\frac{1}{4}\left(x^{3}-3 x^{2} x_{c}+3 x x_{c}^{2}-x_{c}^{3}\right)\right)
$$


Here we use the dimensionless variables defined in the Section $2 ; r=x-x_{c}, x$ and $x_{c}$ are particle and rigid counter beam coordinates at the IP. The first and third addends in Equation (12) depend on $x$ only and determine the linear and nonlinear tune shifts. The second and fourth addends determine the values of the dipole and sextupole external perturbations. The last two addends can be neglected because $x_{c}<1$. To do a rough estimate of the parametric excitement we can leave the fourth addend only. Then we have

$$
\delta p \approx 3 \pi \xi x^{2} x_{c} .
$$

The counter bunch motion is coherent on the time scale $\lambda^{-1}$ (i.e., $2 / g$ turns). As the emittance increase can occur due to random kicks, we can only consider that independent kicks Equation (13) follow one after an other in time $\lambda^{-1}$. In this case the emittance growth rate per turn due to quadrupole noise of the counter beam is equal to

$$
\left(\frac{d \varepsilon}{d n}\right)_{N L} \approx \frac{1}{2}\left\langle\delta p^{2}\right\rangle \frac{g}{2}
$$

Taking into account that

$$
\left\langle x_{c}^{2}\right\rangle=\frac{1}{2 g} \Delta^{2},
$$

and for a Gaussian function distribution

$$
<x^{4}>=3,
$$

substituting Equations (13), (15) and (16) in Equation (14), we finally have an additional contribution to the emittance growth rate per turn due to nonlinear excitement

$$
\left(\frac{d \varepsilon}{d n}\right)_{N L} \approx \frac{27 \pi^{2}}{8} \xi^{2} \Delta^{2}
$$

Note that this additional contribution is not damped by the feedback system and does not depend on gain $g$. For small $g$ this addend gives much smaller emittance growth than kicks of magnets and can be neglected. But it is important that in the case of a large gain $(g>0.1)$ it decreases the emittance growth suppression.

The results of simulations are presented in Figure 8. One can see that for a small value of $g$ there is a small difference in the emittance growth rates for the cases when the rigid beam can or cannot move in transverse directions. For large values of $g$ there is a great difference in the emittance growth rates. The difference between the emittance growth rates of both cases is in good agreement with the estimate of Equation (17). Finally, using Equations (51) of Reference 1, and Equations (8) and (17), we can write down the formula for the emittance growth rate

$$
\frac{d \varepsilon}{d t} \approx \frac{A \xi^{2}}{g^{2}}\left[\left(\frac{d \varepsilon}{d t}\right)_{0}+\frac{f_{0} g^{2}}{2 \beta}<x_{\text {noise }}^{2}>\right], \quad A=\left(3.3+67 g^{2}\right), \quad \xi<g \leq 0.4
$$




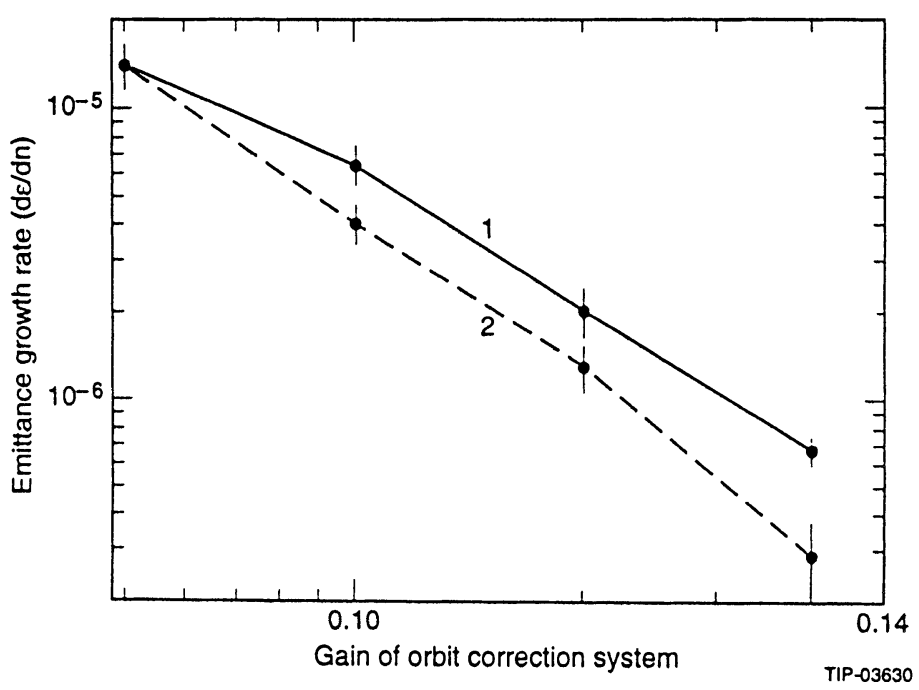

FIGURE 8: The dependence of the emittance growth rate on the dimensionless gain $g$ for the cases with (upper curve) and without (lower curve) coherent motion of the rigid beam. $\Delta=0.0316, \xi=0.005, \nu=0.78$. The number of turns is equal to 7000 .

whose predictions are in a good agreement with simulation results (within the accuracy of the simulation). Here $\left\langle x_{\text {noise }}^{2}\right\rangle$ is the beam position monitor (BPM) resolution (noises of the feedback system referenced to the BPM sensitivity), $\beta$ is the $\beta$-function at BPM location, and $f_{0}$ is the revolution frequency. In the definition of $A$, the first addend is determined by the simulation results without coherent effects presented in Section 2.2, and the second addend $27 \pi^{2} / 4 \approx 67$ is determined by Equation (18).

All results shown below are for $g=0.2$ because further increase of the gain does not produce a significant damping of the emittance growth.

\section{INFLUENCE OF THE BEAM SEPARATION ON THE EMITTANCE GROWTH}

For the first hadron colliders (SppS and Tevatron) the aiming of the beams at each other at the IP was done automatically due to charge symmetry; dedicated electrostatic separators were used to separate beams in arcs. But for colliders such as the SSC where the two beams are confined in different rings, additional efforts should be made for this aiming. It is well known that in the electron-positron colliders even a small beam separation $(\sim 0.1-0.2 \sigma)$ can drastically increase the beam-beam effects and decrease the luminosity. ${ }^{6}$ Taking into account the very small beam size at the IP $(\sigma \approx 3-5 \mu m)$, we should set very rigid requirements for this aiming $(0.3-1 \mu \mathrm{m})$. 


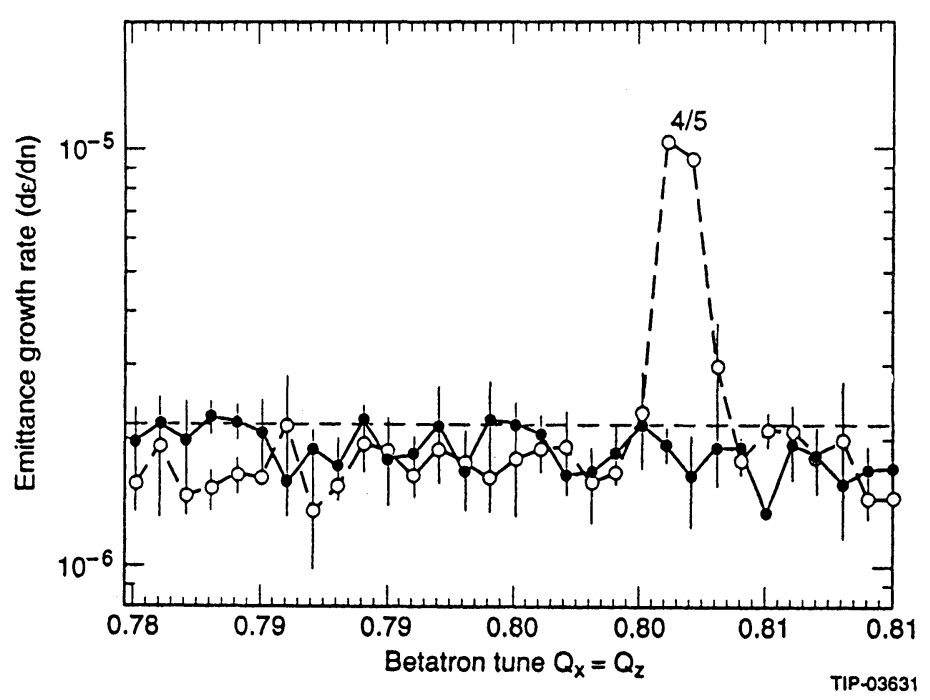

FIGURE 9: The dependence of the emittance growth rate on the betatron tune for zero (solid curve) and $0.5 \sigma$ (dashed curve) beam separation; with coherent motion of the rigid bunch, $g=0.2, \Delta=0.0316, \xi=0.005$. The dotted line is plotted using Equation (8) with $A=7$. The number of turns is equal to 5000 .

Figure 9 shows the dependence of the emittance growth rate versus the betatron tune for zero and $0.5 \sigma$ separations. One can see that the beam separation excites the strong $4 / 5$ odd resonance whose influence was suppressed by the interaction symmetry in the zero separation case. At points located far enough from resonances, the emittance growth rate is smaller for the $0.5 \sigma$ separation case.

The dependence of the emittance growth rate on the separation at the IP for the constant tune equal to 0.78 is shown in Figure 10. One can see that in this case (nonresonant) the emittance growth rate decreases with a separation increase. This very important feature determines that in the case of large hadron colliders (when $\xi$ value is much smaller than in the case of electron-positron colliders) we can choose the collider tune far from resonances and there will not be any decrease in luminosity due to the beam-beam effects. In this case the main limitation on the beam separation is determined by a luminosity decrease due to the geometrical beam separation

$$
L=L_{0} \exp \left(-\frac{\delta x^{*^{2}}}{4 \sigma^{*^{2}}}\right),
$$

and the requirements of the beam aiming are much less restrictive. Here $\delta x^{*}$ and $\sigma^{*}$ denote the beam separation and the rms beam size at the IP. From Equation (19) it follows that a separation of $0.6 \sigma$ produces a drop of $10 \%$ in the luminosity. 


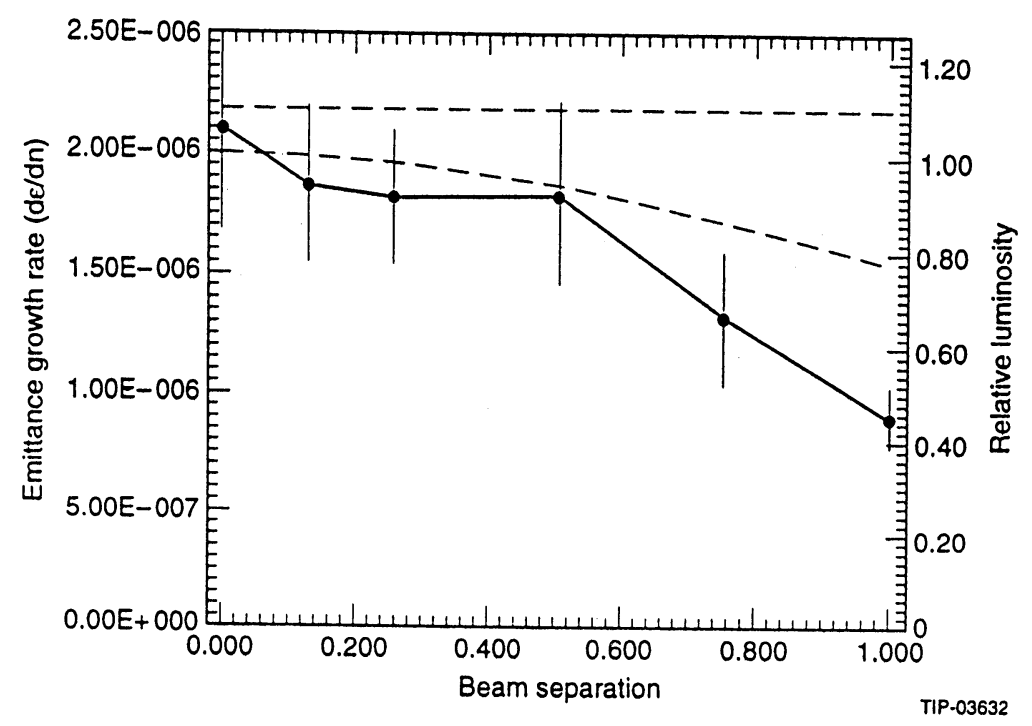

FIGURE 10: The dependences of the emittance growth rate (solid curve) and collider luminosity (dashed curve) on the beam separation at the IP; with coherent motion of the rigid bunch, $g=0.2, \Delta=0.0316$, $\xi=0.005$, and $\nu=0.78$. The upper dotted line is plotted using Equation (8) with $A=7$. The number of turns is equal to 5000 .

For comparison, the dependence of the luminosity on the separation, in accordance with Equation (19), is also shown in Figure 10.

\section{INFLUENCE OF THE NOISE SPECTRAL DENSITY ON THE EMITTANCE GROWTH}

In the simulation described previously, the sequential beam kicks were independent, which corresponds to the white noise approximation. In reality, the spectral density of external noises decreases rapidly with increasing frequency. Thus, for ground motion the spectral density at frequency $1 \mathrm{~Hz}$ is ten orders higher than at the SSC first resonance frequency of $760 \mathrm{~Hz}$ (see Figure 1 of Reference 1). In this case, the issue of low frequency influence on the emittance growth rate should be addressed. In particular, a few articles 7,8 were published that predict a strong influence of low frequencies on emittance growth. To clarify this issue we carried out simulations for two kinds of spectral density. In the first case the spectral density is proportional to $1 / \omega^{2}$, and in the second case the harmonic perturbation is added to the white noise. 


\subsection{Computer Model for the $1 / \omega^{2}$ Noise}

To simulate noise with the required spectral density, the algorithm of the generation of the random variables $\zeta^{\left(p_{x}\right)}, \zeta^{\left(p_{y}\right)}, \zeta^{(x)}$, and $\zeta^{(y)}$ in Equation (3) has been changed from that described in Section 2.1. In this case, the kick value on turn $(n+1), \tilde{\zeta}_{(n+1)}$ has been computed as the sum of the kick value $\tilde{\zeta}_{n}$ on the previous turn and a random number $\zeta$, so that $\tilde{\zeta}_{n+1}=\zeta+\tilde{\zeta}_{n}$. In other words, the kick value on turn $n$ is proportional to the sum of random numbers

$$
\tilde{\zeta}_{n}=\sum_{k=1}^{n} \zeta_{k}, \quad\left\langle\zeta_{k}^{2}\right\rangle=1,
$$

where $\zeta_{k}$ are random numbers with Gaussian distribution. For the sake of brevity, we omitted here $x$ and $p$ superscripts which are used in Equation (21). Thus, instead of Equation (3), the following transformation has been used:

$$
\begin{array}{ll}
p_{x_{j(n+1)}}=p_{x_{j n}}+\Delta_{s} \tilde{\zeta}_{n}^{\left(p_{x}\right)}, & x_{j(n+1)}=x_{j n}+\Delta_{s} \tilde{\zeta}_{n}^{(x)}, \\
p_{y_{j(n+1)}}=p_{y_{j n}}+\Delta_{s} \tilde{\zeta}_{n}^{\left(p_{y}\right)}, & y_{j(n+1)}=y_{j n}+\Delta_{s} \tilde{\zeta}_{n}^{(y)}
\end{array}
$$

where $\Delta_{s}$ is the strength of the noise, $n$ numerates turns, and $j$ numerates particles. Strictly speaking, the sequence of random numbers in Equation (20) does not represent a stationary process because its correlation function depends on time. However, we believe that it models the real ground motion for which the integral of spectral density diverges on the low limit.

To introduce the spectral density of the process described in Equation (20), let us consider a function $f(t)$ which is equal to a series of $\tilde{\zeta}_{n}$ at fixed moments of time $t_{n}=n T$, when a bunch goes through the point of perturbation. Here $T$ is the revolution period. Although the function $f(t)$ does not describe the stationary process, we can consider its spectral density on interval $\left[0, T_{s}\right]$ as an average:

$$
S(\omega)=\frac{\left\langle\left|f_{\omega}^{2}\right|\right\rangle}{T_{s}}, \quad f_{\omega}=\frac{1}{\sqrt{2 \pi}} \int_{0}^{T_{s}} f(t) e^{t \omega t} d t,
$$

whose limit for $T_{s} \rightarrow \infty$ will approach the general spectral density definition of Equation (9) of Reference 1. As shown in Appendix C, as defined by Equation (22), the spectral density of the process Equation (20) for $T_{s} \rightarrow \infty$ is equal to

$$
S(\omega)=\frac{1}{2 \pi T \omega^{2}} .
$$

As can be seen from Equation (21), to model displacements of a large number of quadrupoles we simultaneously produce a change of beam momentum and coordinate at the IP after each turn in a way similar to that described in Section 2.1.

The defined random function has diffusive character - which means that its rms value grows in time as $\propto t^{1 / 2}$. This results in a beam separation at the IP that grows in time by the same way. To suppress this beam separation, we have used an additional slow feedback system which models a closed-orbit correction system at a real collider. 
This additional system operates together with the fast one described above: we call it the correction system. The system changes the momentum and position of the beam (in both transverse planes) at the IP according to the formula:

$$
p_{n+1}=p_{n}-g_{s} \sum_{k=1}^{n} x_{k}, \quad x_{n+1}=x_{n}+g_{s} \sum_{k=1}^{n} p_{k} .
$$

Such a correction system can be easily realized in a real storage ring by the integration of signals that are proportional to transverse beam displacements. For the computer simulation, it is important that this system strongly damps a slow, closedorbit displacement and produces a negligible effect on high-frequency motion. As an illustration of the correction system operation, we mention here that for $g_{s}<g$ in the case when the beam is driven to oscillate with the low frequency $\omega$ and amplitude $\Delta_{s}$ by an external force, turning on the slow feedback system suppresses the amplitude of the oscillation down to the value

$$
\Delta_{s}\left(2 \pi \frac{\omega}{\Omega g_{s}}\right)
$$

which prevents the beam separation at the IP. Here $\Omega$ is the revolution frequency.

The beam motion is stable if

$$
0 \leq \frac{g_{s}}{\tan (\pi \nu)} \leq g
$$

which usually does not limit simulations where $g_{s}$ is always much smaller than $g$. As can be seen from Equation (26), in order to ensure stability of the motion, $g_{s}$ has to be positive for the working points below a half-integer resonance and negative for these above a half integer resonance. All our simulations were performed with negative $g_{s}$. For brevity we will omit the sign of $g_{s}$ below. To simplify simulations, the kicker of the correction system was also located just after the IP. Both rigid and soft beams were influenced by the described feedback systems and noise.

Neglecting the influence of the correction system on betatron oscillations, one can obtain from Equations (18) and (23) the emittance growth rate per turn for the case when both described noises (the white noise and the $1 / \omega^{2}$ noise) affect the beam

$$
\frac{d \varepsilon}{d n} \approx \frac{A \xi^{2}}{2 g^{2}}\left(\Delta^{2}+\frac{\Delta_{s}^{2}}{2 \pi^{2}} \sum_{k=-\infty}^{\infty} \frac{1}{(\nu-k)^{2}}\right),
$$

where $A=A(g)$ was determined from simulations with the white noise see Equation (18) and $\Delta_{s}$ and $\Delta$ are values of the white and $1 / \omega^{2}$ noises.

\subsection{Simulation Results for the $1 / \omega^{2}$ Noise}

The dependence of the emittance growth and value of coherent motion on the correction system damping $g_{s}$ are shown in Figure 11. One can see that although the amplitude of coherent motion strongly depends on the damping $g_{s}$, the emittance growth 


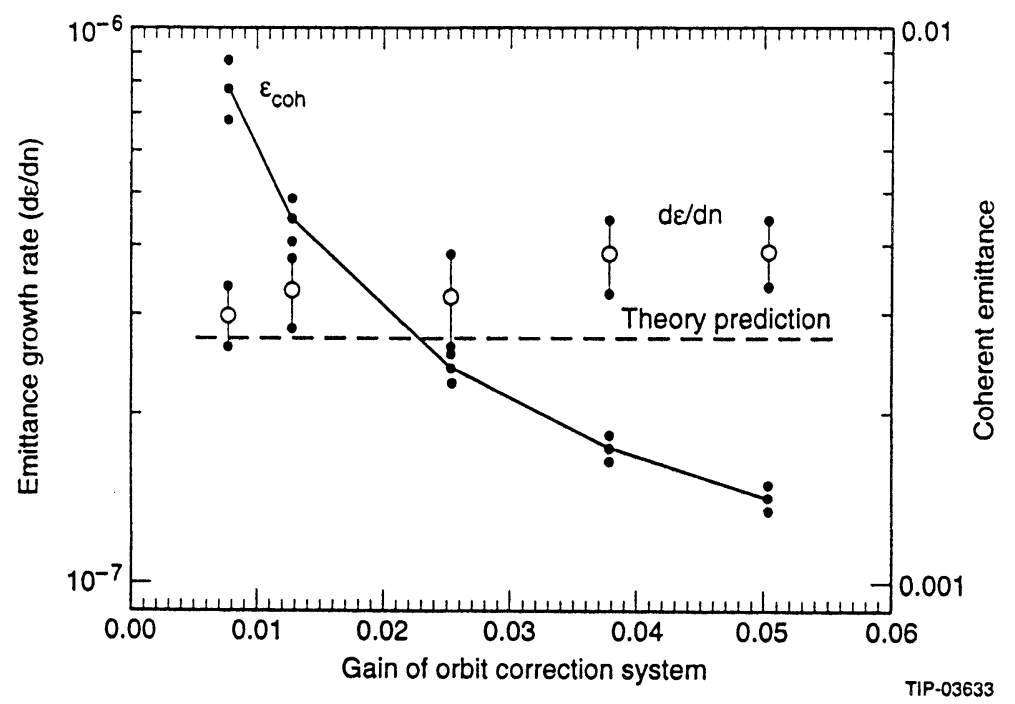

FIGURE 11: The dependencies of coherent motion value (solid curve) and the emittance growth rate $(\bigcirc)$ on the gain of orbit correction system with coherent motion of the rigid bunch and $1 / \omega^{2}$ noise; $g=0.2$, $\Delta_{s}=0.0103, \xi=0.005$, and $\nu=0.78$. The dotted line is plotted using Equation (27) with $A=7$. The number of turns is equal to 5000 .

rate does not depend on $g_{s}$, within the accuracy of the simulation, and coincides with theoretical predictions based on the study of emittance growth under white noise influence. A small increase in the emittance growth rate for a large value of damping $g_{s}$ is associated with lowering of the decrement of high-frequency motion due to an interference of the feedback and the correction systems. As follows from Equation (26), the stability of motion will be lost for $g_{s}>0.165$. Figure 11 clearly demonstrates that there is no direct connection between the emittance growth and the value of coherent motion and that the emittance growth rate is determined by noise spectral density on resonance frequencies. This is also demonstrated by Equation (51) of Reference 1 and Equation (27).

To test the agreement of simulation results with theoretical predictions, we carried out scanning of betatron tunes. The results of the simulations are shown in Figure 12. One can see a good coincidence between simulations results and the theoretical predictions for all working points located far enough from resonances. ${ }^{c}$ Some inconsistency arises for betatron tunes close to integer resonance. This is due to the interference of fast and slow feedback systems which decreases the damping decrement

\footnotetext{
${ }^{c}$ One can see two points in the vicinity of resonance of $4 / 7$ and $5 / 7$ drop down to values much smaller than the theory predictions. We have done an additional simulation with larger staistics for these points and found that this drop is of a random character.
} 


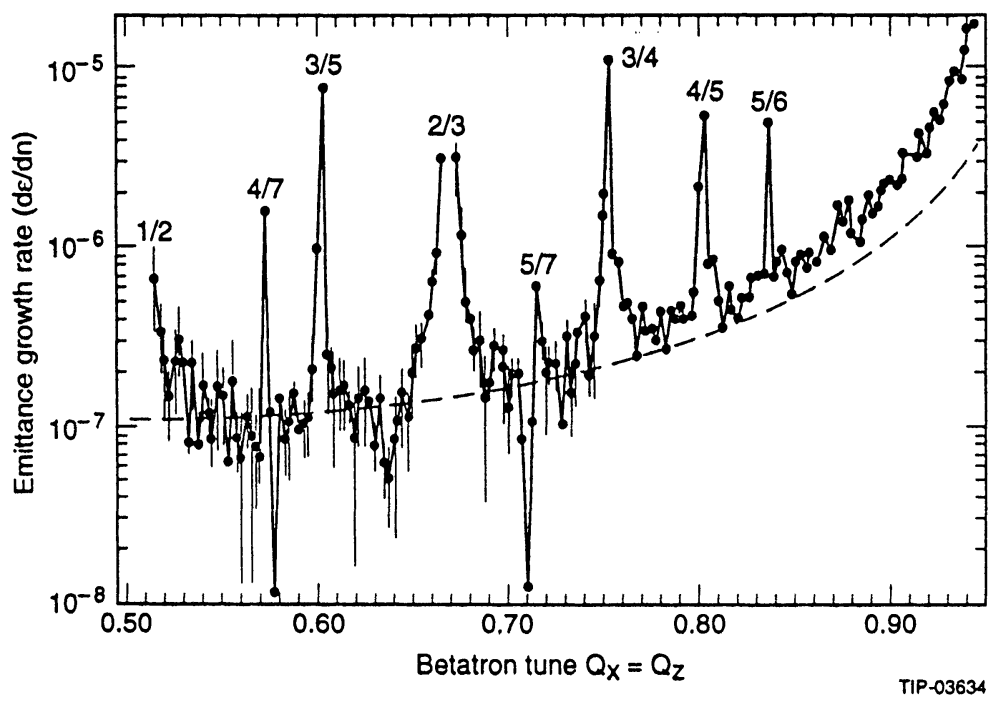

FIGURE 12: The dependence of the emittance growth rate on tune with coherent motion of the rigid bunch and $1 / \omega^{2}$ noise; $g=0.2, \Delta=0, \Delta_{s}=0.0103, \xi=0.005$, and $\nu=0.78$. The dotted line is plotted using Equation (27) with $A=7$. Number of turns is equal to 5000 .

and for given parameters determines the stability loss for $0.9604<\nu<1(g=0.2$, $\left.g_{s}=0.025\right)$. One can see also that the low-frequency motion strongly increases the influence of odd resonances, much in the same way as beam separation at the IP. For comparison, the results of simulation for white noise with the same level of coherent motion are shown in Figure 13. One can see that in this case, all odd resonances are strongly suppressed, and the emittance growth rate does not depend on the tune for all non-resonant points.

All previous simulations were carried out for equal betatron tunes $\nu_{x}=\nu_{y}$ i.e., on coupling resonance. From the common point of view, it should not produce a difference in emittance growth for the working points located at or outside the coupling resonance because of the equality of transverse emittances. Nevertheless, in order to test this, we also scanned the tune in the direction transverse to the coupling resonance along the straight line between points with coordinates the in tune space of $(0.78,0.78)$ and $(0.81,0.75)$. One can see from Figure 14 that for all non-resonant tunes the emittance growth rate is the same as on the coupling resonance. The two resonant peaks are associated with resonances of $\nu_{x}=4 / 5$ and $\nu_{y}=3 / 4$. As it should be for the colliding beams with equal particle charges, the resonances are shifted to the side of higher betatron frequencies.

It follows from the general definition of the Fourier transform that for a finite time interval $T_{s}$ the lowest frequency is equal to $1 / T_{s}$. Simulations were done for a 


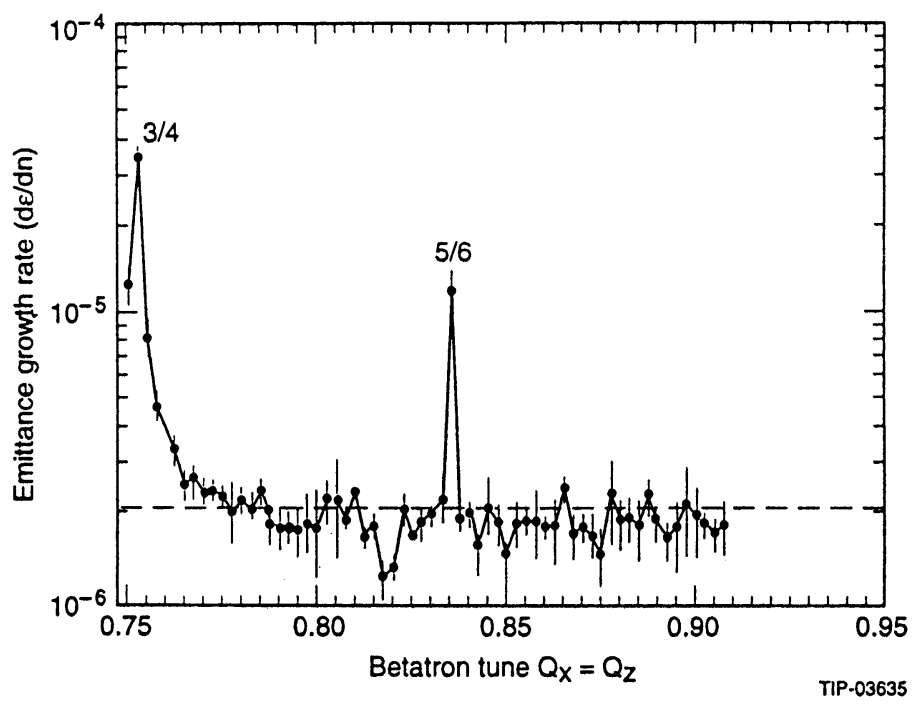

FIGURE 13: The dependence of the emittance growth rate on tune with coherent motion of the rigid bunch and white noise; $g=0.2, \Delta=0.0316, \Delta_{s}=0, \xi=0.005$, and $\nu=0.78$. The dotted line is plotted using Equation (27) with $A=7$. Number of turns is equal to 5000 .

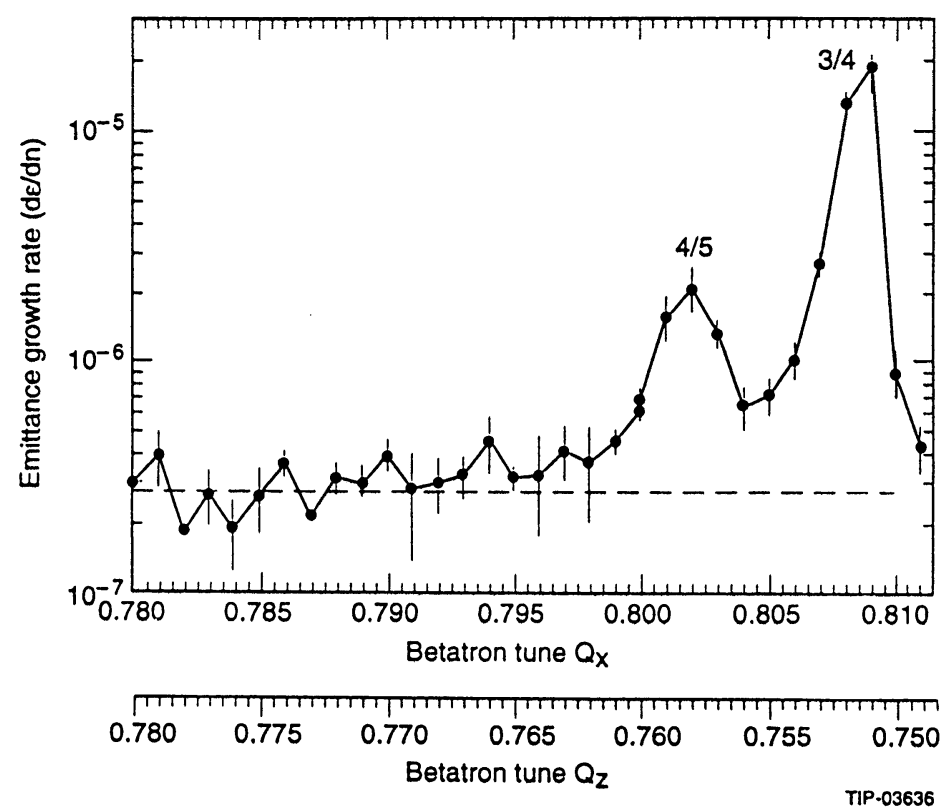

FIGURE 14: The dependence of the emittance growth rate on tune for the scan in the direction transverse to the coupling resonance with coherent motion of the rigid bunch; $g=0.2, g_{s}=0.025, \Delta=0, \Delta_{s}=0.0103$, and $\xi=0.005$. The dotted line is plotted using Equation (27) with $A=7$. The number of turns is equal to 5000 . 
comparatively small number of turns $\left(N_{\text {turn }} \leq 8000\right)$, which means that the minimal frequency of the perturbation is equal to $f_{0} / N_{\text {turn }}\left(\approx 0.5 \mathrm{~Hz}\right.$ for the SSC). For the $1 / \omega^{2}$ noise the simulations determined that the spectral density at the lowest frequency is in $\left([\nu] N_{\text {turn }}\right)^{2}$ times larger than at the lowest betatron frequency $[\nu] f_{0}$. In other words, the difference between the maximum spectral density and the spectral density determining the emittance growth is an order of $10^{6}-10^{7}$ times. Although the simulations verified that the theory developed in Reference 1 is applicable for $1 / \omega^{2}$ noise, for frequencies higher than $\left(10^{-3}-10^{-4}\right) f_{0}$ it seems insufficient for real applications associated with ground motion because its spectral density increases with decreasing frequency as $1 / \omega^{3}-1 / \omega^{4}$ on a frequency range $1-2000 \mathrm{~Hz} \cdot{ }^{10}$ It gives difference in spectral densities at 1 and $1000 \mathrm{~Hz}$ about $\approx 10^{9}-10^{10}$. Thus, a further development of the simulations should be done to test the applicability of the developed theory for larger variations of spectral density.

\subsection{The Simulations with Harmonic Perturbation}

As was said before for the case without the feedback system, the real width of a resonance is determined by the betatron tune spread. This is not evident for the case with ultimate damping, when $g$ is close to 1 , because the beam coherent motion has a wide frequency band of order $g \Omega$. Nevertheless, it seems plausible that the emittance growth occurs only due to a motion inside the bunch, and from the general point of view, the beam response frequency band has to be equal to the betatron tune spread.

To test this, we carried out the simulation for external excitement consisting of two parts: the random (white) noise and harmonic perturbation of beam horizontal momentum

$$
\begin{aligned}
& p_{x}^{\prime}=p_{x}+\frac{\Delta}{\sqrt{2}} \zeta^{\left(p_{x}\right)}+a \cdot \cos \left(2 \pi \nu_{e} n\right), \quad x^{\prime}=x+\frac{\Delta}{\sqrt{2}} \zeta^{(x)}, \\
& p_{y}^{\prime}=p_{y}+\frac{\Delta}{\sqrt{2}} \zeta^{\left(p_{y}\right)}, \quad y^{\prime}=y+\frac{\Delta}{\sqrt{2}} \zeta^{(x)} .
\end{aligned}
$$

Only the soft beam was influenced by the additional harmonic perturbation. The rigid beam was influenced by the white noise, as before.

The results of the simulations are presented in Figures 15 and 16. One can see that the harmonic perturbation produces an additional emittance growth only in a resonance band with width equal to the betatron tune spread $\xi f_{0}$. Outside of the resonance band there is not any influence within the accuracy of the simulations.

To estimate roughly the emittance growth rate in resonance, we can consider that the harmonic excitement spectral density is averaged on the frequency band of an order of the betatron tune spread; then the spectral density of the kick is ${ }^{d}$

$$
S(\omega) \approx \frac{a^{2}}{2 \xi \Omega} .
$$

\footnotetext{
${ }^{d}$ The factor $1 / 2$ appears because the spectral density has two peaks, one in positive and another one in negative frequencies.
} 


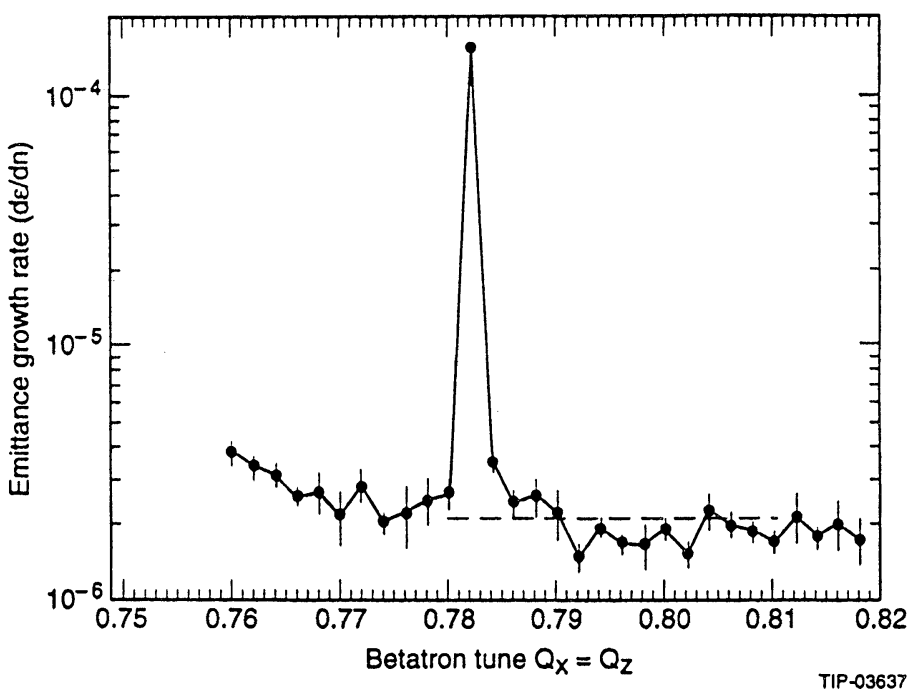

FIGURE 15: The dependence of the emittance growth rate on tune with additional white noise harmonic excitement and coherent motion of the rigid bunch; $g=0.2, a=0.0948, \Delta=0.0316, \xi=0.005$, and $\nu_{e}=0.78$. The dotted line is plotted using Equation (8) with $A=7$. The number of turns is equal to 5000 .

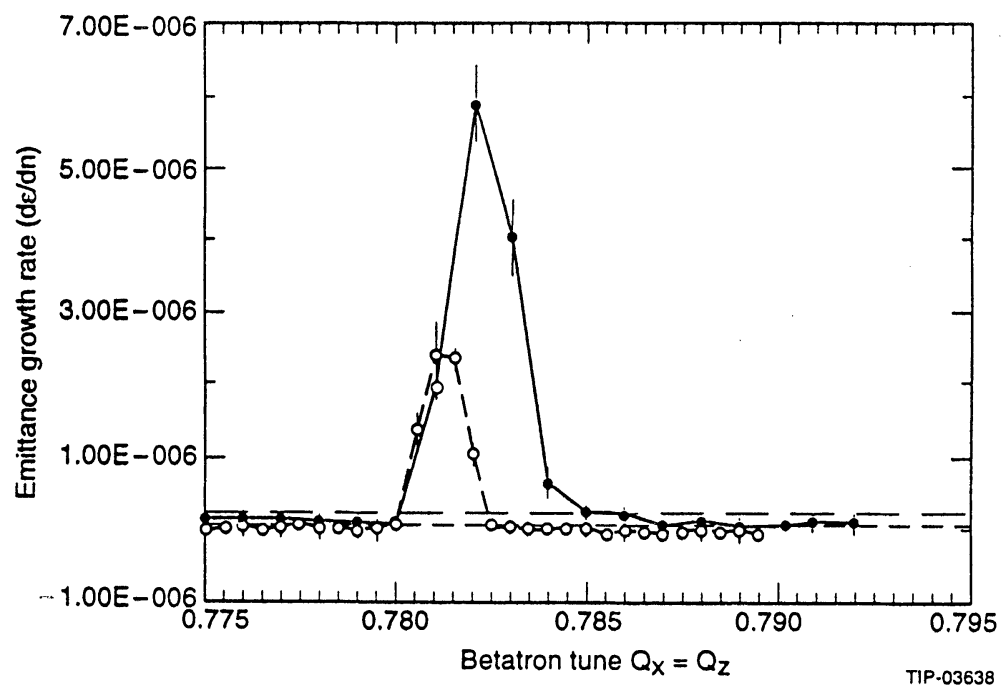

FIGURE 16: The dependence of the emittance growth rate on tune with additional white noise harmonic excitement and coherent motion of the rigid bunch; $g=0.2, a=0.01, \Delta=0.01, \nu_{e}=0.78$. The solid curve $\xi=0.005$, the dashed $\xi=0.0025$, the straight dotted lines are plotted using Equation (18) with $A=7$. The number of turns is equal to 5000 . 
Substituting this expression in the Equation (18) determining the emittance growth rate, one finally has

$$
\frac{d \varepsilon}{d t} \approx B \frac{A \xi}{4 g^{2}} a^{2} .
$$

Here, the constant $B$ should be of order 1 . The results presented in Figure 16 determine $B \approx 0.25$. This figure clearly demonstrates that the emittance growth rate in the resonance (within the accuracy of the simulation) is proportional to the $\xi$ value as was predicted by Equation (30).

Note that, in fact, the process of emittance growth is much more complicated than it has been presented here. It is associated with large inhomogeneities of the distribution function (considered in particle tune space) in the vicinity of the perturbation frequency. Therefore, both the estimate and the simulation results are very rough. To produce more accurate numerical simulations, we need to increase the number of turns from 10000 to some millions and increase the particle numbers in the same relation in order to suppress the stochastic cooling effects. This requires more than $10^{6}$ extra calculations and goes beyond the capability of modern computers. Nevertheless, in expected SSC conditions, the level of the feedback system noise will be much larger than an amplitude of harmonic perturbation. Additionally, the slow-frequency motion will produce permanent fluctuations of particle tunes. These effects will strongly smooth inhomogeneities in the distribution function that, in the end, justifies the estimate.

\section{DEPENDENCE OF THE EMITTANCE GROWTH ON BEAM SEPARATION AT PARASITIC INTERACTION POINTS}

To obtain maximum luminosity, many bunches are used in the super collider. In this case, besides the main IP, there are a lot of parasitic collisions at long-range IPs before the beams are separated into different storage rings. Thus, the important question is: how large does the beam separation at long-range collision points have to be?

We have studied this question for the SSC collider. In this case, the beam has long range collisions every $2.5 \mathrm{~m}$ for almost $100 \mathrm{~m}$ before the beams are separated into different rings. The value of beam separation in the parasitic IPs expressed by the rms size changes comparatively little. ${ }^{e}$ Because of the large value of the $\beta$ function in long-range IPs, the phase advance between them is small in comparison to the phase advance between the main and any parasitic IP whose value is about $\left(\pi / 2-\beta^{*} / L_{n}\right)$. Here $\beta^{*}$ is the $\beta$-function at the main IP, and $L_{n}$ is the distance between the main IP and a long-range IP. In this case, in the first approximation, one can replace all longrange collisions by two, one located on the left and one on the right side of the main IP with the betatron phase advance equal to $\pm \pi / 2$. To suppress a closed-orbit distortion,

\footnotetext{
${ }^{e}$ Note that the value of beam separation, expressed in $\sigma$, does not change between the IP and the first quad and changes slightly inside of the final focus quads because of their focusing.
} 


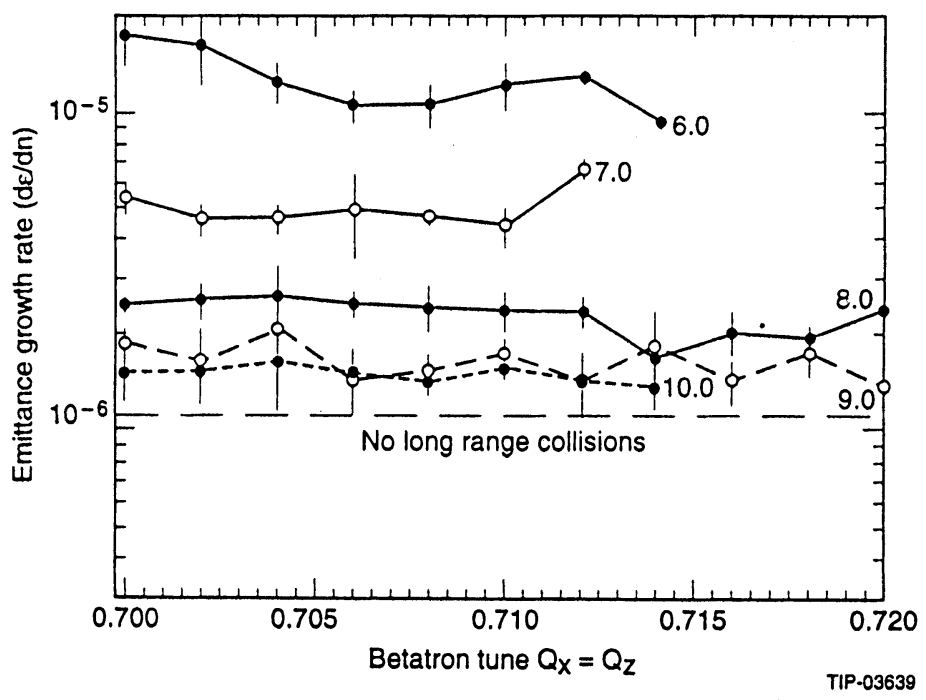

FIGURE 17: The dependence of the emittance growth rate on tune for different beam separations in the long-range IPs with the coherent motion of the rigid bunch; $g=0.2, \Delta=0.0316, \xi=0.0036$, and $N_{c l}=32$. The dotted lines are plotted using Equation (8) with $A=7$. The number of turns is equal to 5000 .

the dipole component of kick is subtracted from the kick value so that the value of kick at one global long-range collision point is

$$
\delta \vec{p}_{L N_{j}}=8 \pi \xi N_{c l}\left(\frac{\vec{R}_{j}}{R_{j}^{2}}\left(1-e^{R_{j}^{2} / 2}\right)-\frac{\vec{\delta}}{\delta^{2}}\left(1-e^{\delta^{2} / 2}\right)\right), \quad \vec{R}=\vec{r}_{j}-\vec{r}_{0}-\vec{\delta}
$$

Here $\vec{\delta}$ is beam separation at long range collisions, $\vec{r}_{j}, \vec{r}_{0}$ are a particle and the rigid beam positions related to the closed orbits, and $N_{c l}$ is a number of long-range collision points. Because of the crossing angle, the value of beam separation at the second global long-range collision point changes sign.

The results of simulations are presented in Figure 17. One can see that for the SSC case $\left(N_{c l} \approx 32\right)$ even at beam separation of $8 \sigma$, the emittance growth rate is more than two times larger than without parasitic collisions. Note that in the case of long-range collisions, the nonlinearity increases rapidly with betatron amplitude. It produces very strong resonance excitement of particles with large amplitudes, i.e., the beam-beam effects in the long-range IPs will strongly increase diffusion in the beam halo.

The maximum baseline value of the SSC beam crossing angle is equal to $67 \mu \mathrm{rad}$ (half of full crossing value) which corresponds to beam separation in the range of $(10-14) \sigma$ for $\beta^{*}=0.5 \mathrm{~m}$. It follows from the simulation results that this is close to the 
minimum value of the crossing angle acceptable from the beam-beam effects point of view. Further increasing of the crossing angle will decrease the luminosity due to the geometric factor as

$$
\mathbf{L}=\mathbf{L}_{0}\left(1+\frac{1}{2}\left(\frac{\alpha \sigma_{s}}{\sigma^{*}}\right)^{2}\right)^{-1 / 2} .
$$

Here $\alpha$ is the crossing angle (half of a full one), $\sigma_{s}$ is the rms bunch length, and $\sigma^{*}$ is the rms beam size at the IP. For $\alpha=67 \mu \mathrm{rad}, \sigma_{s}=6 \mathrm{~cm}$, and $\sigma^{*}=5 \mu \mathrm{m}$, one gets $\mathbf{L} / \mathbf{L}_{0}=0.78$ that is not negligible.

\section{DISCUSSION}

The main conclusion that follows as the result of this work is that the transverse emittance grows only under the influence of resonance frequencies, i.e., frequencies that are within the betatron frequency spread and its sidebands $f_{n}=f_{0}(\nu-n)$. Although the spectral density at low frequencies $\left(f<f_{0}\right)$ is much larger, these frequencies do not produce emittance growth with time. ${ }^{f}$

The experimental study of the beam-beam effects at proton-antiproton colliders has shown that the measured $\xi$ values are much smaller than those for the electronpositron colliders and usually do not surpass 0.005 for one IP. The main difference between the proton-antiproton and electron-positron colliders is that for the former there is no damping of separate particle motion. In this case the effect of beam-beam interactions can be very strongly affected by the external noise which produces an additional emittance smear. Our results show that for small enough $\xi$ and for the working point located far enough from strong resonances, one can neglect a very complicated picture of the motion and consider the external noise as the main source of the emittance growth.

Our simulations show that the emittance growth rate for tunes located far enough from resonances can be determined with a good accuracy $(\approx 30 \%)$ by using the expression

$$
\frac{d \varepsilon}{d t} \approx \xi^{2}\left(\frac{3.3}{g^{2}+3.3 \xi^{2}}+67\right)\left[\left(\frac{d \varepsilon}{d t}\right)_{0}+\frac{f_{0} g^{2}}{2 \beta_{1}}<x_{\text {noise }}^{2}>\right], \quad g \leq 0.5, \quad \xi \ll 0.1,
$$

where $(d \varepsilon / d t)_{0}$ is determined by Equations (13) and (19) and the spectral density in Equation (13) of Reference 1 has to be averaged on the frequency band equal to the betatron tune spread. This expression is the interpolation of the two limiting cases, one in which the feedback system strongly damps the emittance growth $g>\xi$ (compare

\footnotetext{
$f$ It does not mean that there are no limitations on the frequency noises. In particular, it was shown theoretically and experimentally ${ }^{10}$ that low frequency pertubation in the current of quadrupoles will confine the dynamic aperture of the storage ring. Nevertheless, it should be noted that it does not produce an emittance growth in the sense that is discussed here.
} 
TABLE 1: Main Parameters of Hadron Colliders

\begin{tabular}{|c|c|c|c|c|}
\hline & SppS & Tevatron & LHC & SSC \\
\hline Energy [GeV] & 315 & 900 & 8000 & 20000 \\
\hline Circumference $[\mathrm{km}]$ & 6.93 & 6.29 & 26.66 & 87.12 \\
\hline Revolution frequency [kHz] & 43.3 & 47.7 & 11.25 & 3.44 \\
\hline Betatron tunes: $\nu_{x}$ & 26.685 & 19.405 & 71.28 & 123.78 \\
\hline$\nu_{y}$ & 26.675 & 19.415 & 70.31 & 123.78 \\
\hline Number of IPs, $N_{\mathrm{IP}}$ & 3 & 12 & 3 & 4 \\
\hline \multicolumn{5}{|l|}{ Head-on beam-beam tune shift $(p / \bar{p})$} \\
\hline per collision $\xi_{x}\left[10^{-3}\right]$ & $5.0 / 5.3$ & $1.5 / 2.1$ & 3 & 0.9 \\
\hline$\xi_{y}\left[10^{-3}\right]$ & $3.3 / 3.3$ & $1.5 / 2.1$ & 3 & 0.9 \\
\hline \multirow{2}{*}{$\begin{array}{ll}\text { summed } & N_{\mathrm{IP}} \xi_{x}\left[10^{-3}\right] \\
& N_{\mathrm{IP}} \xi_{y}\left[10^{-3}\right]\end{array}$} & $15 / 16$ & $18 / 25$ & 10 & 3.6 \\
\hline & $11 / 11$ & $18 / 25$ & 10 & 3.6 \\
\hline Normalized emittance $\varepsilon_{n}(p / \bar{p})[\mu \mathrm{m}]$ & $3 / 1.7$ & $4 / 2$ & 3.75 & 1 \\
\hline Rms emittance $\varepsilon=\varepsilon_{n} / \beta \gamma(p / \bar{p})[\mathrm{nm}]$ & $8.9 / 5.2$ & $4.2 / 2.1$ & 0.46 & 0.047 \\
\hline The first resonance frequencies & 13.6 & 19.3 & 3.1 & 0.76 \\
\hline$f=f_{0}(\nu-n), \quad[\mathrm{kHz}]$ & 29.6 & 28.4 & 8.0 & 2.68 \\
\hline Luminosity life time [hour] & 24 & 24 & 10 & 24 \\
\hline$\beta$-function at BPM location [m] & 40 & 50 & 200 & 390 \\
\hline Required BPM resolution ${ }^{g}[\mu \mathrm{m}]$ & 0.33 & 0.16 & 0.9 & 1.2 \\
\hline
\end{tabular}

with Equation (18) herein) and the other in which the feedback system influence is negligible $g>\xi$ (compared with Equation (19) of Reference 1).

A strong influence of external noise on the beam lifetime was demonstrated at the Tevatron. ${ }^{11,12}$ Experiments show that the emittance grows rapidly with decrease of the fractional part of the collider tune. The main aim of the experiments was to decrease the fractional part of the collider tune so as to change it from the normal collider tune (fractional part $~ 0.4$ ) to a tune in the range of $0.06-0.10$. It was expected that this would decrease the emittance growth rate because of a smaller density of nonlinear resonances at the new working point. However, the emittance growth rate was strongly increased. The main reason for this growth is that the spectral density of external perturbation grows rapidly with frequency, decrease and this generates a larger emittance growth.

\footnotetext{
${ }^{g}$ Required BPM resolution was calculated with the use of Equation (18) for $(d \varepsilon / d t)_{0}=0$, when the gain $g$ is canceled in the equation.
} 
All present hadron colliders (i.e., SppS, Tevatron) have worked without transverse feedback system during the collision experiments because of strong emittance growth which was generated by the feedback system itself. This was possible due to the small beam intensity. The new generation of super colliders (SSC, LHC), with their higher ultimate luminosity, requires as large beam current as possible, and this requires powerful longitudinal and transverse feedback systems. As it was shown here, a good transverse feedback system should not produce the emittance growth but must strongly damp it. Thus, to get the ultimate luminosity required by super colliders, a new level of the feedback system technology has to be reached. The comparison of the main parameters of the colliders that already exist and are under design are shown in Table 1. As can be seen, for all installations the required BPM resolution has to be better than $1 \mu \mathrm{m}$.

The results of the work lead to some important practical recommendations:

1. As the transverse emittance growth is due to the resonance frequencies only and the spectral density of external noise decreases very rapidly with increasing frequency, it is important to choose the collider tunes as far as possible from the integer resonances. Note that quadrupole noise produces much smaller emittance growth than dipole noise and in most cases can be neglected. For the SSC case, reducing the collider tune from 0.78 to 0.59 permits a decrease in the emittance growth of 3-10 times.

2. A strong feedback system, with a damping time of only a few turns, allows one to decrease the emittance growth by more than 2-3 orders of magnitude. In this case, the main obstacles to the full damping of the beam heating are the beam-beam effects which lead to the betatron frequency spread in the beam and consequently to emittance smear and growth. It is also important to have a high resolution beam position monitor in the feedback system $(0.2-0.5 \mu \mathrm{m})$.

3. Obtaining acceptable values of the emittance growth depends-both on the vibroclimate in the collider tunnel and on the mechanical properties of magnets and their supports which can considerably amplify vibrations in case of poor design.

4. It is important to note that for a given amount of external noise, an acceptable $\xi$ value determined by the beam-beam effects increases proportionally to the emittance square root, $\varepsilon^{1 / 2}$. This means that the suggestion in Reference 4 of coalescing ten bunches into one will increase the luminosity $10^{1 / 2} \sim 3$ times for the same noise and beam current. To have the same strength of the beam-beam effects, the beam transverse emittance has also to be increased 10 times.

\section{ACKNOWLEDGEMENTS}

I should like to thank V. Parkhomchuk and V. Shiltsev for fruitful discussions at the beginning of this work and G. Stupakov for the close collaboration in the theoretical study of the emittance growth and the great help in preparing the text. 
I should also like to express my deep gratitude to W. Nexsen and S. Sheynin for their careful reading of this article and a lot of suggestions for the improvement of its language and style.

\section{REFERENCES}

1. V.A. Lebedev, V.V. Parkhomchuk, V.D. Shiltsev, and G.V. Stupakov, "Emittance growth due to noise and its suppression with the feedback system in large hadron colliders." SSCL Preprint 188, Dallas, TX (1993); Particle Accelerators, this issue.

2. V.A. Lebedev, V.V. Parkhomchuk, V.D. Shiltsev, and A.N. Skrinsky, "Suppression of emittance growth caused by mechanical vibrations of magnetic elements in presence of beam-beam effects." Preprint INP 91-120, Novosibirsk (1991).

3. V.V. Parkhomchuk, and A.N. Skrinsky, "Cooling methods of charged particle beams," Physics of Elementary Particles and Atomic Nuclei (Joint Institute for Nuclear Research, Dubna, 1981), 12, 3, p. 557.

4. Site-specific conceptual design of the Superconducting Super Collider (SSCL, July 1990).

5. A. Chao and R. Ruth, "Coherent beam-beam instability in colliding-beam storage rings," Particle accelerators, 16, 201 (1985).

6. A.B. Temnych, "Observation of beam-beam effects on VEPP-4," in the Proceedings of the Third Advanced ICFA Beam Dynamics Workshop on Beam-Beam Effects in Circular Colliders, (Novosibirsk, USSR, 1989).

7. K.-Y. Ng and J.M. Peterson, "Emittance growth due to beam motion," SSCL-441 (May 1991); IEEE Particle Accelerator Conference (San-Francisco, CA, 1991.

8. K.-Y. Ng, "Emittance growth due to a small low frequency perturbation," FN-575 (1991).

9. X. Altuna, et al., "The 1991 dynamic aperture experiment at the CERN SPS," CERN-SL/91-43(AP); LHC Note 171.

10. V.V. Parkhomchuk, V.D. Shiltsev, and H.J.Weaver, "Measurements of the ground motion vibrations at the SSC," SSCL-Preprint-323 (May 1993).

11. R.P. Johnson and P. Zhang, "A new Tevatron collider working point near the integer," in Proceedings of the 1988 IEEE Particle Accelerator Conference, II, 806.

12. P. Zhang, Thesis: "A study of tunes near integer values in hadron colliders," Fermi National Laboratory, Batavia, IL 60510. (1991).

13. G. Lopez, "Head-on and long range beam-beam tune shifts spread in the SSC," SSCL-442 (May 1991).

14. G.V. Stupakov, V.V. Parkhomchuk, V.D. Shiltsev, "Decoherence of a Gaussian beam due to beambeam interaction". SSCL-Preprint-495, 1993. 


\section{APPENDIX A: DEPENDENCE OF BETATRON TUNE SHIFT ON AMPLITUDE DUE TO HEAD-ON BEAM-BEAM EFFECTS}

For a round beam, the kick values of a particle with coordinates $x, y$ at the IP are equal to (see Equation (2))

$$
\delta p_{x}^{\prime}=\frac{8 \pi \xi x}{r^{2}}\left(1-\exp \left(-\frac{r^{2}}{2}\right)\right), \quad x, p_{x} \leftrightarrow y, p_{y} .
$$

We use the same coordinates here as in Section 2. Taking into account that in the first-order of the perturbation theory, the tune shifts are

$$
\Delta \nu_{x}=\frac{1}{2 \pi a_{x}}\left\langle\delta p_{x} \sin \left(\psi_{x}\right)\right\rangle, \quad \Delta \nu_{y}=\frac{1}{2 \pi a_{y}}\left\langle\delta p_{y} \sin \left(\psi_{y}\right)\right\rangle,
$$

we can express tune shift by an integral: ${ }^{13}$

$$
\begin{aligned}
\Delta \nu_{x}\left(a_{x}, a_{y}\right)= & \frac{\xi}{\pi^{2}} \int_{0}^{2 \pi} d \psi_{x} \int_{0}^{2 \pi} d \psi_{y} \frac{\sin ^{2} \psi_{x}}{a_{x}^{2} \sin ^{2} \psi_{x}+a_{y}^{2} \sin ^{2} \psi_{y}} \\
& \left(1-\exp \left(-\frac{a_{x}^{2} \sin ^{2} \psi_{x}+a_{y}^{2} \sin ^{2} \psi_{y}}{2}\right)\right), x \leftrightarrow y,
\end{aligned}
$$

where $a_{x}, a_{y}$ are amplitudes of oscillations and $\psi_{x}, \psi_{y}$ are their phases. We evaluated this integral numerically.

To calculate the mean value $\langle\Delta \nu\rangle$ and mean square value $\left\langle\Delta \nu^{2}\right\rangle$ of the tune shift, one has to integrate the tune shift with function distribution

$$
\begin{aligned}
\langle\Delta \nu\rangle=\langle\Delta \nu\rangle_{x}=\langle\Delta \nu\rangle_{y}= & \int_{0}^{\infty} a_{x} d a_{x} \int_{0}^{\infty} a_{y} d a_{y} \Delta \nu\left(a_{x}, a_{y}\right) \\
& \exp \left(-\frac{a_{x}^{2}+a_{y}^{2}}{2}\right), \\
\left\langle\Delta \nu^{2}\right\rangle=\left\langle\Delta \nu^{2}\right\rangle_{x}=\left\langle\Delta \nu^{2}\right\rangle_{y}= & \int_{0}^{\infty} a_{x} d a_{x} \int_{0}^{\infty} a_{y} d a_{y} \Delta \nu^{2}\left(a_{x}, a_{y}\right) \\
& \exp \left(-\frac{a_{x}^{2}+a_{y}^{2}}{2}\right) .
\end{aligned}
$$


To calculate the dispersion from Equation (A4), one has to use the general equation:

$$
\sqrt{\delta \bar{\nu}^{2}}=\sqrt[i]{\left\langle\Delta \nu^{2}\right\rangle-\langle\Delta \nu\rangle^{2}} .
$$

The numerical calculations determine the following values: ${ }^{14}$

$$
\begin{aligned}
& \sqrt{\delta \bar{\nu}^{2}} / \xi \simeq 0.17, \\
& \langle\Delta \nu\rangle / \xi \simeq 0.63 .
\end{aligned}
$$

If $a_{x}$ (or $a_{y}$ ) is equal to zero, then the integral (A3) can be evaluated analytically:

$$
\delta \nu(a)=\frac{4 \xi}{a^{2}}\left(1-I_{0}\left(\frac{a^{2}}{4}\right) \exp \left(-\frac{a^{2}}{4}\right)\right) .
$$

We used this expression in the numerical simulations described in Section 2.2. The numerical averaging of Equation (A7) with two dimensional Gaussian function distribution

$$
f(a)=\frac{a}{2} \exp \left(-\frac{a^{2}}{4}\right)
$$

determines the average tune shift and its dispersion:

$$
\begin{aligned}
& \sqrt{\delta \bar{\nu}^{2}} / \xi \approx 0.224, \\
& \langle\delta \nu\rangle / \xi \approx 0.624 .
\end{aligned}
$$

which values are close to the more rigorous result of Equation (A6).

\section{APPENDIX B: COHERENT BEAM-BEAM EFFECTS IN THE PRESENCE OF EXTERNAL NOISE IN THE HARD BUNCH MODEL}

To estimate the influence of the coherent beam-beam effects on the emittance growth, we will consider a simple linear model in an assumption of rigid bunches and one IP. Because of linearity we can consider a one-dimensional task. In this case after an interaction, the bunch changes its momentum by

$$
\delta p_{1}=4 \pi \xi(x-1-x-2), \quad 1 \leftrightarrow 2 .
$$

Here the dimensionless variables are the same as those used in Section 2, indices 1 and 2 correspond to the two colliding beams, and $\xi$ is the linear head-on beam-beam tune shift. 
For two colliding bunches one can write the matrix of transition through the IP ;

$$
X^{\prime}=M_{I P} X \equiv\left|\begin{array}{cccc}
1 & 0 & 0 & 0 \\
4 \pi \xi & 1 & -4 \pi \xi & 0 \\
0 & 0 & 1 & 0 \\
-4 \pi \xi & 0 & 4 \pi \xi & 1
\end{array}\right| \cdot\left|\begin{array}{c}
x_{1} \\
p_{1} \\
x_{2} \\
p_{2}
\end{array}\right|
$$

and through the whole ring

$$
X_{n+1}=M X_{n}=M_{R} M_{I P} X_{n} \equiv\left|\begin{array}{cccc}
c & s & 0 & 0 \\
-s & c & 0 & 0 \\
0 & 0 & c & s \\
0 & 0 & -s & c
\end{array}\right| \cdot\left|\begin{array}{cccc}
1 & 0 & 0 & 0 \\
4 \pi \xi & 1 & -4 \pi \xi & 0 \\
0 & 0 & 1 & 0 \\
-4 \pi \xi & 0 & 4 \pi \xi & 1
\end{array}\right| \cdot\left|\begin{array}{l}
x_{1_{n}} \\
p_{1_{n}} \\
x_{2_{n}} \\
p_{2_{n}}
\end{array}\right|
$$

where both the storage rings have equal tunes $\nu_{1}=\nu_{2}=\nu$, and

$$
c=\cos 2 \pi \nu, \quad s=\sin 2 \pi \nu .
$$

Let the beam be influenced by random kicks:

$$
\vec{\Delta}_{n}=\left|\begin{array}{l}
\delta x_{1_{n}} \\
\delta p_{1_{n}} \\
\delta x_{2_{n}} \\
\delta p_{2_{n}}
\end{array}\right|
$$

where

$$
\left\langle\delta x_{1_{n}}^{2}\right\rangle=\left\langle\delta p_{1_{n}}^{2}\right\rangle=\left\langle\delta x_{2_{n}}^{2}\right\rangle=\left\langle\delta p_{2_{n}}^{2}\right\rangle=\frac{\sigma^{2}}{2}
$$

which corresponds to the fluctuations of $N$ lenses $(N>1)$ distributed along the circumference and moved with rms displacements equal to $\sigma / N^{1 / 2}$. After $n$ turns, the beam coordinates will be:

$$
X_{n}=\sum_{k=1}^{n} M^{n-k} \vec{\delta}_{k}=\sum_{k=1}^{k} \sum_{i=1}^{4} a_{k l} V_{i} \Lambda_{i}^{n-k}
$$


Here $V_{i}$ and $\Lambda_{i}$ are the eigen-vectors and the eigen-numbers of transition matrix

$$
M=\left|\begin{array}{cccc}
c+4 \pi \xi s & s & -4 \pi \xi s & 0 \\
4 \pi \xi c-s & c & -4 \pi \xi c & 0 \\
-4 \pi \xi s & 0 & c+4 \pi \xi s & s \\
-4 \pi \xi c & 0 & 4 \pi \xi c-s & c
\end{array}\right|
$$

and the coefficients $a_{k i}$ are defined by the equation

$$
\vec{\Delta}_{k}=\sum_{t=1}^{4} a_{k i} V_{i} .
$$

The rms beam deviation from the closed orbit after $n$ turns is equal to:

$$
\begin{gathered}
\left\langle x_{n}^{2}\right\rangle=\frac{1}{4}\left\langle\left(X_{n}^{*}, X_{n}\right)\right\rangle=\frac{1}{4} \sum_{k=1}^{n} \sum_{i=1}^{4} \sum_{l=1}^{n} \sum_{j=1}^{4}\left\langle a_{k i}^{*} \Lambda_{i}^{*^{n-k}} a_{l j} \Lambda_{j}^{n-k}\left(V_{i}^{*}, V_{j}\right)\right\rangle \\
=\frac{1}{4} \sum_{i=1}^{4} \sum_{i=1}^{4}\left\langle a_{i}^{*} a_{j}\right\rangle\left(V_{i}^{*}, V_{j}\right) \sum_{k=1}^{n} \Lambda_{i}^{*^{n-k}} \Lambda_{j}^{n-k}=\frac{n}{4} \sum_{i=1}^{4}\left\langle a_{i}^{*} a_{i}\right\rangle\left(V_{i}^{*}, V_{i}\right)
\end{gathered}
$$

where ${ }^{*}$ is a sign of complex conjugation, and it was taken into account that

$$
\left\langle a_{k i}^{*} a_{l j}\right\rangle=\left\langle a_{i}^{*} a_{j}\right\rangle \delta_{k l}
$$

and that only members with $i=j$ make a contribution which is proportional to $n$ because $\Lambda_{i}^{*^{n-k}} \Lambda_{i}^{n-k}=1$.

After a simple calculation one can find that the eigenvectors and eigen-numbers of the transition matrix $\mathrm{M}$ are equal to:

$$
\begin{gathered}
\lambda_{1,2}=c \pm i s \equiv e^{ \pm 2 \pi i \nu}, \quad \lambda_{3,4}=c+4 \pi \xi s \pm \sqrt{1-(c+4 \pi \xi s)^{2}} \equiv e^{ \pm i \delta}, \quad \\
V_{1,2}=\left|\begin{array}{c}
1 \\
\pm i \\
1 \\
\pm 1
\end{array}\right|, \quad V_{3,4}=\left|\begin{array}{c}
1 \\
-4 \pi \xi \pm i \sqrt{1-(c+4 \pi \xi s)^{2}} \\
4 \pi \xi \mp i \sqrt{1-(c+4 \pi \xi s)^{2}}
\end{array}\right|=\left|\begin{array}{c}
1 \\
\left(c-e^{\mp i \delta}\right) / s \\
1 \\
-\left(c-e^{ \pm i \delta}\right) / s
\end{array}\right|
\end{gathered}
$$


and the scalar multiplications of the eigenvectors are:

$$
\begin{gathered}
\left(V_{i}, V_{j}\right)=\left|\begin{array}{llll}
4 & 0 & 0 & 0 \\
0 & 4 & 0 & 0 \\
0 & 0 & p & q \\
0 & 0 & q^{*} & p
\end{array}\right|, \\
p=\frac{4}{S^{2}}(1-c \cos \delta), \quad q=\frac{2}{s^{2}}\left(1+e^{-2 i \delta}-2 c e^{-i \delta}\right) .
\end{gathered}
$$

The solution of Equation (B9) determines the coefficients $a_{i k}$

$$
a_{k l}=\frac{1}{4}\left(\vec{\Delta}_{k}, V_{1}^{*}\right), \quad a_{k 2}=\frac{1}{4}\left(\vec{\Delta}_{k}, V_{2}^{*}\right), \quad a_{k 4}=a_{k 3}^{*}=\frac{p\left(\vec{\Delta}_{k}, V_{4}^{*}\right)-q\left(\vec{\Delta}_{k}, V_{3}^{*}\right)}{p^{2}-q q^{*}} .
$$

Substituting Equations (B14) and (B16) in Equation (B10) and averaging:

$$
\left\langle a_{1}^{*} a_{1}\right\rangle=\left\langle a_{2}^{*} a_{2}\right\rangle=\frac{1}{8} \sigma^{2}, \quad\left\langle a_{3}^{*} a_{3}\right\rangle=\left\langle a_{4}^{*} a_{4}\right\rangle=\frac{p}{p^{2}-q q^{*}} \frac{\sigma^{2}}{2},
$$

one finally has the emittance growth rate

$$
\frac{d \varepsilon}{d n}=\frac{\sigma^{2}}{4}\left(1+\frac{p^{2}}{p^{2}-q q^{*}}\right)=\frac{\sigma^{2}}{4}\left(1+\frac{(4 \pi \xi)^{2}}{2 s\left[s-4 \pi \xi c-(4 \pi \xi)^{2} s\right]}\right) .
$$

The second addend in brackets is connected with the contribution of the coherent dipole interaction. It tends to infinity near the boundary of stability determined by the following equation: ${ }^{5}$

$$
s-4 \pi \xi c-(4 \pi \xi)^{2} s=0 .
$$

\section{APPENDIX C: SPECTRAL DENSITY OF THE DIFFUSION}

The noise used in the computer model is a sequence of random numbers

$$
\delta_{n}=\Delta_{s} \sum_{k=1}^{n} \zeta_{k}, \quad\left\langle\delta_{n}^{2}\right\rangle=n\left\langle\Delta_{s}^{2}\right\rangle
$$


where $\zeta_{k}$ is random numbers $\left\langle\zeta_{k}^{2}\right\rangle=1$ with the Gaussian distribution. To calculate the spectral density, we have to determine a random function $f(t)$ that is equal to these random numbers in discrete points $t=n T$. Although the spectral density at high frequencies will depend on a function choice, the spectral density at low frequencies and the sum of spectral densities at resonance frequencies (which determines the emittance growth) do not depend on a function choice. Therefore, we determine this function by the simplest way:

$$
f(t)=\Delta_{s} \sum_{k=1}^{\infty} \Theta(t-k T) \zeta_{k}
$$

where

$$
\Theta(t)= \begin{cases}0, & t<0, \\ 1, & t>0,\end{cases}
$$

$\zeta_{k}$ are the random numbers, and $T$ is the revolution time. Because, as previously noted, the calculation of the spectral density for diffusion is not the mathematically correct task, let us consider a simple physical model. If the pendulum is be excited by the force Equation (C2)

$$
\ddot{x}+\omega^{2} x=f(t),
$$

then we can consider that at the moment $t=n T$, the pendulum equilibrium position is changed by $\Delta_{s} \zeta_{n} / \omega^{2}$ which will excite oscillations with the same amplitude. So, after averaging, the rms value of oscillations for large enough $t$ will be

$$
\left\langle x^{2}(t)\right\rangle=\frac{\Delta_{s}^{2} t}{2 T \omega^{2}} .
$$

Note that this value is counted in relation to a new equilibrium position whose value increased as $t^{1 / 2}$. This is directly related to real physics measurements using gauges such as seismometers, accelerometers, etc., which are not sensitive to zero and small frequencies.

On the other hand, it is well known that the pendulum rms displacement for stationary function (correct task) increases as

$$
\left\langle x^{2}(t)\right\rangle=\frac{\pi t}{\omega^{2}} S(\omega), \quad S(\omega)=S(-\omega),
$$

where $S(\omega)$ is the spectral density of function $f(t)$. Comparing Equation (C5) and Equation (C6) one finally has the spectral density of the diffusion process described by function Equation (C1)

$$
S(\omega)=\frac{\Delta_{s}^{2}}{2 \pi T \omega^{2}}
$$




\section{APPENDIX D: MOTION UNDER INFLUENCE FAST AND SLOW FEEDBACK SYSTEMS}

To analyze stability of beam motion under the influence of the fast feedback system and the orbit correction system let us consider the transfer matrix per turn

$$
\left|\begin{array}{l}
x \\
p
\end{array}\right|_{n+1}=\left|\begin{array}{cc}
c & s \\
-s & c
\end{array}\right|\left[\left|\begin{array}{cc}
1 & 0 \\
0 & 1-g
\end{array}\right| \cdot\left|\begin{array}{l}
x \\
p
\end{array}\right|_{n}+g_{s}\left|\begin{array}{cc}
0 & 1 \\
-1 & 0
\end{array}\right| \cdot \sum_{i=1}^{n}\left|\begin{array}{l}
x \\
p
\end{array}\right|\right],
$$

where $c=\cos (2 \pi \nu), s=\sin (2 \pi \nu)$ and we take into account Equations (1), (4) and (24). We will look for a solution as

$$
V_{n}=\lambda^{n} V_{0},
$$

where $V_{0}$ is the eigenvector and $\lambda$ eigennumber. After summing in the right side of the expression (D1), one has

$$
\left[\left|\begin{array}{ll}
\lambda & 0 \\
0 & \lambda
\end{array}\right|-\left|\begin{array}{cc}
c & s \\
-s & c
\end{array}\right|\left[\left|\begin{array}{cc}
1 & 0 \\
0 & 1-g
\end{array}\right|+\frac{g_{s} \lambda}{\lambda-1}\left|\begin{array}{cc}
0 & 1 \\
-1 & 0
\end{array}\right|\right]\right] V_{0}=0 .
$$

Calculation of the determinant of the transition matrix in Equation (D3) gives the dispersion equation

$$
\lambda^{2}-2 \lambda c\left(1-\frac{g}{2}\right)+(1-g)+g-s \frac{2 \lambda^{2} s}{\lambda-1}+\frac{g_{s}^{2} \lambda^{2}}{(\lambda-1)^{2}}=0 .
$$

This equation has four roots. For small enough values $g$ and $g_{s}$, the two roots are close to betatron motion eigen-numbers $\lambda_{0}$ and $\lambda_{0}^{*}$ :

$$
\lambda_{0}=c+i s,
$$

and the other two roots are close to 1 .

The calculations in the first-order perturbation theory determine that the first two roots are equal:

$$
\lambda_{1}=\lambda_{2}^{*} \approx \lambda_{0}+\frac{i}{2 s}\left(\lambda_{0} c g-g+\frac{g_{s} \lambda^{3 / 2} s}{i \sin (\pi \nu)}\right) .
$$

Taking into account that motion will be stable if $|\lambda|<1$ we have the condition of motion stability

$$
\frac{g_{s}}{\tan (\pi \nu)}<g
$$

The second two roots are

$$
\lambda_{3}=\lambda_{4}^{*} \approx 1-\frac{g_{s}}{(2-g) \sin (\pi \nu)}\left(\cos (\pi \nu) \pm i \sqrt{\sin ^{2}(\pi \nu)-\frac{g}{2}}\right),
$$


and the stability condition is

$$
\frac{g_{s}}{\tan (\pi \nu)}>0
$$

Thus, the particle motion will stable if both conditions Equations (D7) and (D9) are fulfilled.

The analyzed system strongly damps slow frequency external perturbations. To calculate the value of damping, we can add to Equation (D1) an external force

$$
\left|\begin{array}{l}
x \\
p
\end{array}\right|_{n+1}=\left|\begin{array}{cc}
c & s \\
-s & c
\end{array}\right|\left[\left|\begin{array}{cc}
1 & 0 \\
0 & 1-g
\end{array}\right| \cdot\left|\begin{array}{l}
x \\
p
\end{array}\right|_{n}+g_{s}\left|\begin{array}{cc}
0 & 1 \\
-1 & 0
\end{array}\right| \cdot \sum_{i=1}^{n}\left|\begin{array}{l}
x \\
p
\end{array}\right|_{i}\right]+\left|\begin{array}{l}
0 \\
a
\end{array}\right| e^{i \delta n},
$$

where

$$
\delta=\frac{2 \pi \omega}{\omega_{0}},
$$

$\omega$ is frequency of the external force, and $a$ is the force amplitude. We will look for the solution in form of Equation (D2), that gives an equation:

$$
\left|\begin{array}{cc}
c-\lambda-C s & s(1-g)+c C \\
-s-c C & c(1-g)-\lambda-C s
\end{array}\right|\left|\begin{array}{l}
\nu_{1} \\
\nu_{2}
\end{array}\right|=\left|\begin{array}{l}
0 \\
a
\end{array}\right|, \quad C=\frac{g_{s} \lambda}{\lambda-1} .
$$

The solution of the equation has a very simple form for small frequencies $\omega<\omega_{0}$ when $C>1$. In this case, we have a value of coherent emittance equal to

$$
\varepsilon_{c o h}=\frac{1}{2}\left(\nu_{1}^{2}+\nu_{2}^{2}\right) \approx \frac{1}{2}\left(\frac{2 \pi \omega}{\omega_{0} g_{s}} a\right)^{2},
$$

and as one can see, it is strongly damped for small frequencies. 